\title{
Geometry of enstrophy and dissipation, grid resolution effects and proximity issues in turbulence
}

\author{
IVÁN BERMEJO-MORENO ${ }^{1} \dagger$, D. I. PULLIN ${ }^{1}$ \\ AND KIYOSI HORIUTI ${ }^{2}$ \\ ${ }^{1}$ Graduate Aeronautical Laboratories, 205-45, \\ California Institute of Technology, Pasadena, CA 91125, USA \\ ${ }^{2}$ Department of Mechano-Aerospace Engineering, Tokyo Institute of Technology, \\ Tokyo 152-8552, Japan
}

(Received 5 June 2008 and in revised form 20 October 2008)

We perform a multi-scale non-local geometrical analysis of the structures extracted from the enstrophy and kinetic energy dissipation-rate, instantaneous fields of a numerical database of incompressible homogeneous isotropic turbulence decaying in time obtained by DNS in a periodic box. Three different resolutions are considered: $256^{3}, 512^{3}$ and $1024^{3}$ grid points, with $k_{\max } \bar{\eta}$ approximately 1,2 and 4 , respectively, the same initial conditions and $R e_{\lambda} \approx 77$. This allows a comparison of the geometry of the structures obtained for different resolutions. For the highest resolution, structures of enstrophy and dissipation evolve in a continuous distribution from blob-like and moderately stretched tube-like shapes at the large scales to highly stretched sheetlike structures at the small scales. The intermediate scales show a predominance of tube-like structures for both fields, much more pronounced for the enstrophy field. The dissipation field shows a tendency towards structures with lower curvedness than those of the enstrophy, for intermediate and small scales. The $256^{3}$ grid resolution case $\left(k_{\max } \bar{\eta} \approx 1\right)$ was unable to detect the predominance of highly stretched sheet-like structures at the smaller scales in both fields. The same non-local methodology for the study of the geometry of structures, but without the multi-scale decomposition, is applied to two scalar fields used by existing local criteria for the eduction of tube- and sheet-like structures in turbulence, $Q$ and $\left[A_{i j}\right]_{+}$, respectively, obtained from invariants of the velocity-gradient tensor and alike in the $1024^{3}$ case. This adds the non-local geometrical characterization and classification to those local criteria, assessing their validity in educing particular geometries. Finally, we introduce a new methodology for the study of proximity issues among structures of different fields, based on geometrical considerations and non-local analysis, by taking into account the spatial extent of the structures. We apply it to the four fields previously studied. Tube-like structures of $Q$ are predominantly surrounded by sheet-like structures of $\left[A_{i j}\right]_{+}$, which appear at closer distances. For the enstrophy, tube-like structures at an intermediate scale are primarily surrounded by sheets of smaller scales of the enstrophy and structures of dissipation at the same and smaller scales. A secondary contribution results from tubes of enstrophy at smaller scales appearing at farther distances. Different configurations of composite structures are presented. 


\section{Introduction}

The analysis of the rotation- and strain-rate tensor fields, and their scalar counterparts, enstrophy and kinetic energy dissipation, has been recurrent in the study of turbulence through experiments (e.g. Zeff et al. 2003), numerical simulations (e.g. Ishihara et al. 2003) and theoretical developments (e.g. Pullin \& Saffman 1997; He et al. 1998; Wu, Zhou \& Fan 1999). Physically, enstrophy and dissipation correspond to the remaining Galilean-invariant degrees of freedom of fluid particles, rotation and strain, once the dilatation is restricted for incompressible flows. This separation is useful, but it does not decouple the equations of fluid motion. On the contrary, both fields appear in the equations describing the dynamics of each other (Horiuti \& Fujisawa 2008). In fact, the interaction between strain and rotation is intrinsic to the very nature of a three-dimensional turbulence, in particular, vortex-stretching occurs when the strain-rate field stretches and amplifies vorticity.

Turbulence is inherently multi-scale. In the traditional view (Richardson 1922), eddies of large scales break up into smaller ones until viscous effects are dominant and dissipate the energy injected at larger scales. This results in an energy cascade (Richardson 1926; Kolmogorov 1941a,b; Onsager 1945). A complete study of turbulence requires, both in experiments and in numerical simulations, a spatial resolution that resolves the flow up to dissipation scales. A traditional grid resolution criterion used in direct numerical simulations (DNS) of homogeneous turbulence in a periodic box, for example, consists in resolving the flow up to scales of the order of the (average) Kolmogorov scale.

But turbulence is also known to show intermittency (Batchelor \& Townsend 1949; Landau \& Lifshitz 1959; Kolmogorov 1962): fluctuations of flow quantities can reach extreme amplitudes in short intervals of time and spatial distances. Furthermore, fluctuations of different amplitudes tend to cluster. Intermittency increases for higher Reynolds numbers (Okamoto et al. 2007) and also for smaller scales (Brasseur \& Wang 1992). This suggests that the traditional grid resolution criterion, based on an average dissipation scale, might be inappropriate, since much smaller scales are locally present due to those high fluctuations. Therefore, the resolution required to resolve all scales of turbulent flows increases significantly (see Sreenivasan 2004).

These three intimately related properties of turbulence (strain-rotation interaction, multi-scale energy cascade and intermittency) shape the contents of this paper, explored under a geometrical perspective. An interesting example of the geometrical relations between rotation- and strain-rate fields is the local alignment of the vorticity with the intermediate strain-rate eigendirection, for incompressible homogeneous isotropic turbulence. It was observed first in numerical simulations (Ashurst et al. 1987) and confirmed experimentally (see Tsinober, Kit \& Dracos 1992; Tao, Katz \& Meneveau 2000). Theoretical explanations combine local and non-local arguments (see Jiménez 1992; Nomura \& Post 1998; Hamlington, Schumacher \& Dahm 2008). This geometrical property of turbulence has been utilized in the development of subgrid-scale models for use in numerical simulations.

We focus on a different geometrical aspect of those relations, which is structure based. Methods for the identification of structures in turbulence can be divided into local or non-local, attending to whether the classification of the educed structures considers their spatial extent. Local methods are commonly based on scalar fields derived from invariants of the velocity-gradient tensor or related tensors, that highlight certain physical aspects associated with a particular structure either of turbulent flows or of simpler solutions of the Navier-Stokes equations whose phenomenology is 
extrapolated to turbulence (e.g. Burgers vortex tubes and sheets). Points are classified as belonging to one type of structure according to the local value of the field (e.g. by thresholding). Then, points of a common class can optionally be used to define structures with a spatial extent, but this is done as a secondary step, after the point has been already classified. Examples of local methods are the $Q$-criterion (Hunt, Wray \& Moin 1988) and the $\lambda_{2}$ method (Jeong \& Hussain 1995) used to educed vortex tubes. Non-local methods, however, first define the regions of space that are later classified based on geometrical properties of the structure, considering its spatial extent. Geometrical conclusions are then drawn for the set of educed structures. Examples are the calculation of the fractal dimension of a set of iso-surfaces of a particular scalar field by box-counting methods (Moisy \& Jiménez 2004) and the study of the linear distances of regions formed by an ensemble of grid cells from which the same pair of extremal points of the scalar field is reached (Wang \& Peters 2006).

'Tubes' and 'sheets' have been considered the structural geometric building blocks of turbulence. The majority of identification methods (local and non-local) are devoted to educing those types of structures. Traditionally, more attention has been paid to tube-like structures, but recently considerable interest has put forward the eduction of sheet-like structures (see, for example, Tanaka \& Kida 1993; Horiuti 2001). Local methods targeted at educing tubes and sheets, based on physical principles, resort often to visualization of the regions of joined classified points to assess the geometrical character of those regions. Thus, those local methods could use non-local ones to verify qualitatively and be able to quantify the presence of those anticipated geometries.

The dynamics of sheets and tubes are greatly affected by their own interactions, such as the coalescence and reconnection of approaching vortex tubes and the roll-up of vortex sheets to form vortex tubes resulting from the Kelvin-Helmholtz instability. These interactions among sheets and tubes can be seen as the translation of the strainrotation interaction itself to the structural level of turbulence, and help explain the presence of intermittency and the process of multi-scale energy cascade in turbulence (see Kraichnan 1974; Goto 2008). This has led to the development of models of the fine structure of turbulence that account for this structure interaction, such as the strained-spiral vortex model (Lundgren 1982), and later to subgrid-stress models that can be used in numerical large-eddy simulations (LES) of turbulent flows (Misra \& Pullin 1997). Some geometrical aspects of these interactions among structures have also been studied. For example, the prevailing alignment between vorticity and the intermediate eigendirection of the strain-rate tensor is observed to switch towards the direction associated with the most negative eigenvalue of the strain-rate tensor at the ends of tube-like structures (Nomura \& Post 1998), consistent with the compressive straining of the vorticity occurring in those regions. For the incompressible flow, the trace of the strain-rate tensor is null, ensuring at least one positive and one negative eigenvalue of that tensor. Other geometrical analyses regarding the proximity of different types of structures, in relation to their shapes, could be useful in explaining further those interactions and also improve structure-based models of the fine scales of turbulence.

In this paper, we use a numerical database of incompressible homogeneous isotropic turbulence obtained by DNS. In $\S 2$, we perform a multi-scale study of the geometry of educed structures of enstrophy and dissipation-rate fields, comparing the results of both fields. For that purpose, we use the methodology introduced in BermejoMoreno \& Pullin (2008), henceforth referred to as BP, consisting in three main 


$\begin{array}{rcccccccc}N & v & R e_{\lambda} & \langle K\rangle & \langle\epsilon\rangle & L & \lambda & \bar{\eta}\left(\times 10^{-3}\right) & k_{\max } \bar{\eta} \\ 256^{3} & 0.00138 & 77.2 & 0.900 & 0.654 & 0.469 & 0.138 & 8.00 & 1.02 \\ 512^{3} & 0.00138 & 76.87 & 0.897 & 0.654 & 0.469 & 0.137 & 8.00 & 2.05 \\ 1024^{3} & 0.00138 & 77.43 & 0.904 & 0.654 & 0.468 & 0.138 & 8.00 & 4.09\end{array}$

TABLE 1. Parameters for the computed cases: grid points, $N$; kinematic viscosity, $v$; Taylor-microscale Reynolds number, $R e_{\lambda}$; average kinetic energy, $\langle K\rangle$; average dissipation rate, $\langle\epsilon\rangle$; integral length scale, $L$; Taylor microscale, $\lambda$; average Kolmogorov length, $\bar{\eta}$; grid resolution criterion, $k_{\max } \bar{\eta}$ (where $k_{\max }$ is the largest dynamically significant wavenumber). From HF.

steps: extraction, characterization and classification of structures. The extraction includes a multi-scale decomposition of the field. The geometrical characterization is based on differential geometry properties, and considers the spatial extent of the structures. The classification step is aided by clustering techniques. The database includes three different grid resolutions, allowing us to study how this parameter affects the geometry of educed structures and the validity of the traditional grid resolution criterion in DNS from a geometrical standpoint. In $\S 3$, we combine the non-local methodology with two local criteria of identification of vortex tubes and sheets in turbulence (Horiuti \& Takagi 2005) that are based on scalar fields obtained from the velocity-gradient tensor. An assessment of the geometries expected from those local criteria is done. In $\S 4$, we introduce a new methodology for the study of the proximity of structures obtained from two different fields, also in terms of geometry and based on non-local measures through area-coverage quantification. We apply this methodology to the pairs of two scalar fields used by the local identification criteria in $\S 3$ and to the enstrophy and dissipation-rate fields, considering the multiscale decomposition performed in $\S 2$.

\section{Geometry of structures of $\omega_{i} \omega_{i}$ and $S_{i j} S_{i j}$}

The numerical database used here is that of Horiuti \& Fujisawa (2008), henceforth referred to as HF. It corresponds to a DNS of incompressible homogeneous isotropic turbulence decaying in time in a cubic domain of side length $2 \pi$, with periodic boundary conditions. We use runs corresponding to $256^{3}, 512^{3}$ and $1024^{3}$ grid points with the same value of the kinematic viscosity, $v$, resulting in a similar Taylormicroscale Reynolds number, $R e_{\lambda} \approx 77$, and in grid resolution criteria, $k_{\max } \bar{\eta}$, of approximately 1,2 and 4, respectively. Moreover, the initial conditions are the same for the three runs, and therefore, they can be used to compare the geometry of flow structures at different resolutions. Additional parameters for each run at the instant of maximum enstrophy are shown in table 1, extracted from HF, where more details of the computational method can be obtained. The three velocity fields at this time instant for the three grid resolutions are the database for the present study.

In this section, we apply the methodology proposed in BP for the study of the geometry of structures in turbulence to the enstrophy and dissipation fields. Local enstrophy is defined as $\omega_{i} \omega_{i}$, while local dissipation is defined as $\epsilon \equiv 2 v S_{i j} S_{i j}$. $S_{i j}$ is the strain-rate tensor and $\omega_{i}$ is the vorticity field, which is related to the rotation-rate tensor, $\Omega_{i j}$, by $\Omega_{i j}=-\epsilon_{i j k} \omega_{k} / 2, \epsilon_{i j k}$ being the Levi-Civita symbol. Thus, $\omega_{i} \omega_{i}=2 \Omega_{i j} \Omega_{i j}$. Strain- and rotation-rate tensors are obtained from the 
velocity-gradient tensor, $\partial u_{i} / \partial x_{j}$, as

$$
S_{i j}=\frac{1}{2}\left(\frac{\partial u_{i}}{\partial x_{j}}+\frac{\partial u_{j}}{\partial x_{i}}\right), \quad \Omega_{i j}=\frac{1}{2}\left(\frac{\partial u_{i}}{\partial x_{j}}-\frac{\partial u_{j}}{\partial x_{i}}\right) .
$$

We will consider the dissipation rescaled by $(2 v)^{-1}$, that is, $S_{i j} S_{i j}$.

From the velocity field in Fourier space, $\mathscr{F} \mathscr{T}\left(u_{i}\right)$, we first compute $\mathscr{F} \mathscr{T}\left(\omega_{i j}\right)$ and $\mathscr{F} \mathscr{T}\left(S_{i j}\right)$ with spectral resolution. Then the discrete inverse Fourier transform is applied to obtain $\omega_{i}$ and $S_{i j}$ in physical space, where the products $\omega_{i} \omega_{i}$ and $S_{i j} S_{i j}$ are computed. This product operation in physical space introduces aliasing errors in both $\omega_{i} \omega_{i}$ and $S_{i j} S_{i j}$, that could have an influence in the geometry of the structures, particularly at the smaller scales of each resolution. To evaluate this effect, we also computed dealiased $\omega_{i} \omega_{i}$ and $S_{i j} S_{i j}$ fields, removing the aliasing error by means of the 3/2-rule. Both aliased and dealiased data were compared, and we did not find the influence of aliasing error to be significant in our geometrical results, in this case. This comparison is presented in Appendix A. The results presented in the main text of the manuscript correspond to the aliased $\omega_{i} \omega_{i}$ and $S_{i j} S_{i j}$ fields. Note that during the computation of the velocity field, $u_{i}$, by the Fourier pseudo-spectral method, aliasing errors were removed (see HF).

\subsection{Description of the methodology}

In summary, the methodology is applied to a scalar three-dimensional field (in this case, $\omega_{i} \omega_{i}$ and $S_{i j} S_{i j}$, independently) at a given instant in time (here, the instant of maximum enstrophy of the DNS) and consists of three main steps: extraction, characterization and classification of structures. The extraction begins with a multiscale decomposition of the scalar field by means of the discrete curvelet transform (see Candès et al. 2005), which provides a finite set of component fields, associated with the different scales present in the original field. Only the sub-band radial filtering in Fourier space is used in this case, for being the field isotropic. Thus, the multiorientation decomposition provided by the curvelet transform is not used in this particular application. It can be of particular interest in the study of anisotropic fields. Then iso-surfaces are obtained for each component field. For periodic domains, as we have presently, those structures intersecting opposite boundaries are reconnected with their periodic continuation.

The characterization of the extracted structures is done in terms of the area-based joint probability density function (jpdf) of two differential-geometry properties (see Koenderink \& van Doorn 1992): the absolute value of the shape index, $S$, and a nondimensionalization of the curvedness, $C$, plus the dimensionless stretching parameter $\lambda$. Essentially, $S$ and $C$ are, up to scaling factors, polar coordinates in the Cartesian space of the surface principal curvatures $\left(\kappa_{1}, \kappa_{2}\right)$

$$
S \equiv\left|-\frac{2}{\pi} \arctan \left(\frac{\kappa_{1}+\kappa_{2}}{\kappa_{1}-\kappa_{2}}\right)\right|, \quad C \equiv \mu \sqrt{\frac{\kappa_{1}^{2}+\kappa_{2}^{2}}{2}},
$$

where $\mu \equiv 3 V / A, V$ and $A$ are the volume and area, respectively, of the closed surface. $\lambda$ is defined as

$$
\lambda \equiv \sqrt[3]{36 \pi} \frac{V^{2 / 3}}{A}
$$

and equals unity for a sphere. The jpdf, $\mathscr{P}(S, C)$, and $\lambda$ define the geometrical signature of the structure. 
(a)

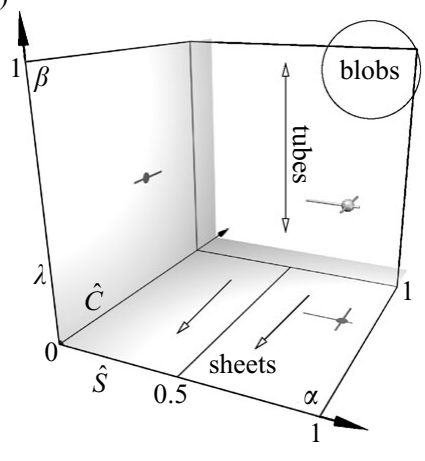

(b)

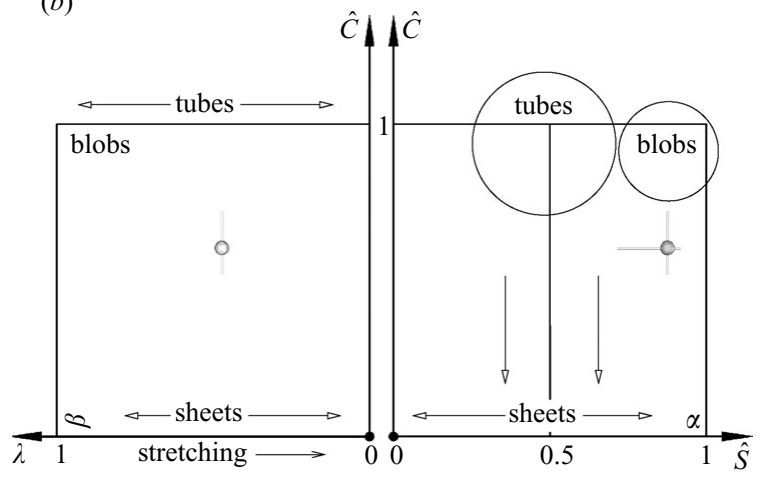

FIGURE 1. Projections of the visualization space with the predominantly blob, tube and sheet-like regions sketched: three-dimensional perspective projection $(a)$, two-dimensional orthogonal projections $(b)$ of the planes $\beta$ (formed by the axes $\hat{C}$ and $\lambda$ ) and $\alpha$ (formed by $\hat{S}$ and $\hat{C}$ ). An example of a glyph consisting of a sphere and four bars along the $\pm \hat{S}$, $\pm \hat{C}$ axes can represent nine parameters of the characterization of the corresponding structure: $\overline{\hat{S}}, \hat{C}, \lambda$ given by the centre of the sphere, upper and lower distances of $S$ and $C$ given by each bar, the surface area $A$ of the associated structure, given by the size of the glyph, and the group to which the structure belongs, given by the colour of the glyph (from BP).

The classification step uses a contracted computational signature consisting of the feature centre $(\hat{S}, \hat{C})$ and the upper and lower distances $\left\{d_{u}^{S}, d_{l}^{S}, d_{u}^{C}, d_{l}^{C}\right\}$ of $\mathscr{P}(S, C)$, defined (see Appendix $C$ of BP) in terms of its first- and second-order moments, plus the stretching parameter, $\lambda$. A three-dimensional visualization space with coordinates $(\hat{S}, \hat{C}, \lambda)$ is defined to represent the geometry of individual structures by means of glyphs. In this application, glyphs are chosen as simple spheres with centre given by $(\hat{S}, \hat{C}, \lambda)$, and whose size and colour correspond to other features of the structure they represent, such as surface area and scale number. More complex glyphs can be chosen to represent additional parameters of the surface. In the visualization space, blob-like structures are located near the region $(\hat{S}, \hat{C}, \lambda) \approx(1,1,1)$; tube-like structures tend to concentrate near the axis $(\hat{S}, \hat{C}, \lambda) \approx(1 / 2,1, \lambda)$ (more stretched for smaller values of $\lambda$ ), while sheet-like structures present small values of both $\hat{C}$ and $\lambda$. See figure 1 for a representation of the visualization space and the limiting geometries (refer to Appendix D of BP for the basis of the location of these limiting geometries). In addition, clustering techniques are applied in a feature space formed by those parameters defining the contracted signature, to educe groups of structures with distinct geometries. Results can also be plotted in the visualization space. This methodology is described in detail in BP.

\subsection{Multi-scale decomposition}

We plot in figure 2 the volume probability density functions (pdfs) ( $a$ ) and the spectra (b), in Fourier space, of the two fields, $\omega_{i} \omega_{i}$ and $S_{i j} S_{i j}$, for the three grid resolutions $\left(256^{3}, 512^{3} 1024^{3}\right)$. It can be observed that the pdf of $\omega_{i} \omega_{i}$ has longer tails than that of $S_{i j} S_{i j}$. This indicates that large amplitude events in the enstrophy field are more prevalent than in the dissipation field (see Chen, Sreenivasan \& Nelkin 1997), and thus, that $\omega_{i} \omega_{i}$ is more intermittent than $S_{i j} S_{i j}$, which is in agreement with results from experiments (Zeff et al. 2003) and numerical simulations (Siggia 1981; Kerr 1985; Chen et al. 1997), using the flatness of each field to measure its intermittency. The discrepancy in the spectra of $\omega_{i} \omega_{i}$ and $S_{i j} S_{i j}$ increases with the wavenumber, $k$, 
(a)

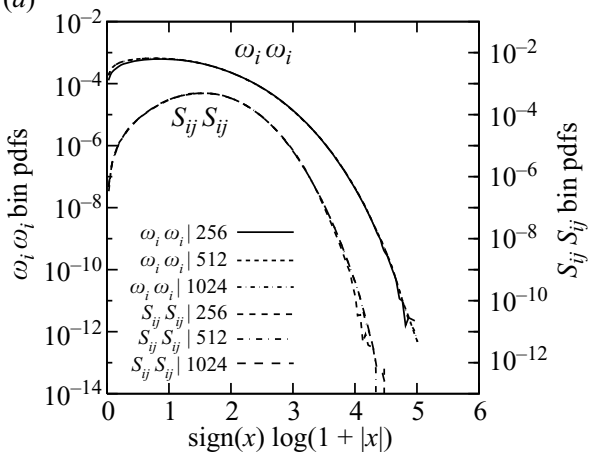

(b)

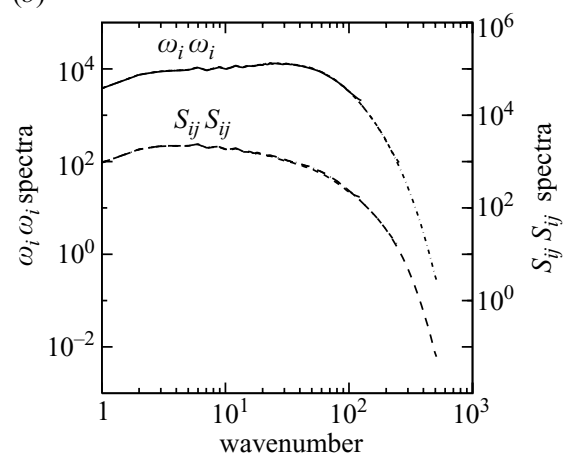

FIGURE 2. Volume pdfs in physical domain $(a)$ and spectra in Fourier domain $(b)$ of $\omega_{i} \omega_{i}$ and $S_{i j} S_{i j}$ fields for the three grid resolutions $\left(256^{3}, 512^{3}\right.$ and $\left.1024^{3}\right)$. Note that the volume pdfs use a transformation of the form $\operatorname{sign}(x) \log (1+|x|)$ in the abscissa coordinate, and that curves for $\omega_{i} \omega_{i}$ and $S_{i j} S_{i j}$ fields use two different vertical axes (both in the pdfs and the spectra), shifted one decade for a clear view (non-intersecting curves).
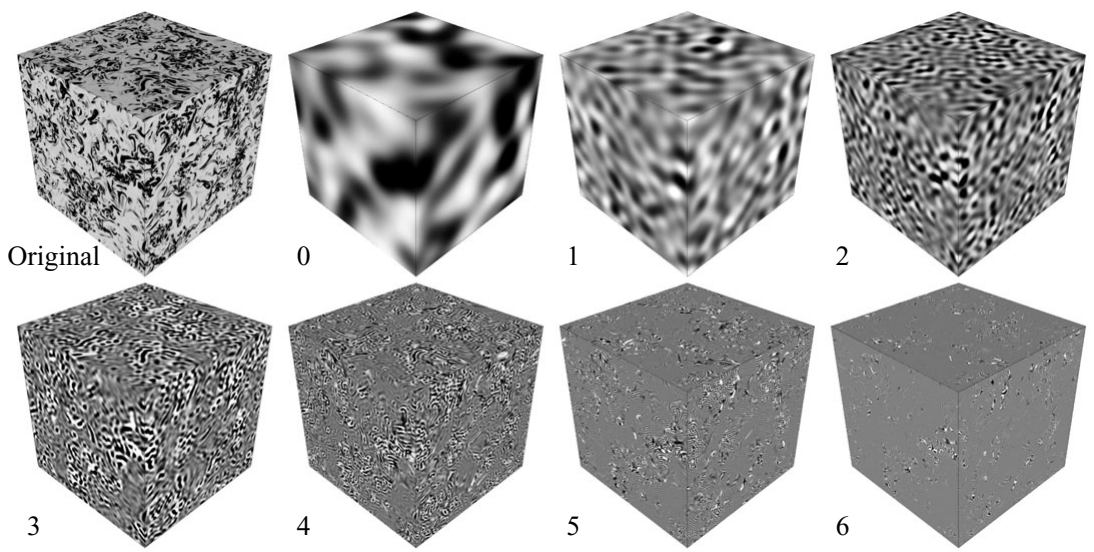

FIGURE 3. Tri-plane cuts of $\omega_{i} \omega_{i}$ and its multi-scale component fields for the $512^{3}$ case.

that is, for smaller scales. This is also in agreement with the results from numerical simulations at higher Reynolds numbers of Ishihara et al. (2003), who found that the maximum difference between spectra of $\omega_{i} \omega_{i}$ and $S_{i j} S_{i j}$ peaks at $k \bar{\eta} \approx 0.4$.

Figure 3 shows the effect in physical space of the multi-scale decomposition for $\omega_{i} \omega_{i}$ with $512^{3}$ grid points, as an example, through plane cuts in the three principal directions of the cubic domain. The original field, containing all scales, is plotted on the top left corner. The rest correspond to each scale number and are obtained after filtering in curvelet domain, returning to physical space.

Volume pdfs (physical domain) and spectra (Fourier domain) of the original and component fields after the multi-scale decomposition are shown in figure 4, for $\omega_{i} \omega_{i}$ $(a)$ and $S_{i j} S_{i j}(b)$ fields in the $1024^{3}$ case. Scales are named by scale numbers from 0 to 7 . Increasing scale numbers, when referred to the component fields in which the original field is decomposed, indicate smaller scales. Thus, 0 corresponds to the largest scale, and 1, 2,.. correspond to smaller and smaller scales. For the $256^{3}$ and $512^{3}$ cases, similar plots (not shown) can be obtained with 5 and 6 as the maximum scale numbers, respectively. Note how, for both $\omega_{i} \omega_{i}$ and $S_{i j} S_{i j}$, the range of the pdfs 


\begin{tabular}{cccccccccc} 
Field & Original & Scale 0 & 1 & \multicolumn{1}{c}{2} & 3 & 4 & 5 & 6 & 7 \\
$\omega_{i} \omega_{i}$ & 5.0 & 41.67 & 20.83 & 9.62 & 4.81 & 2.60 & 1.36 & 0.76 & 0.41 \\
$S_{i j} S_{i j}$ & 20.83 & 41.67 & 20.83 & 10.42 & 5.21 & 2.66 & 1.37 & 0.71 & 0.40
\end{tabular}

TABLE 2. Wavelength at which the spectra of the original fields $\left(\omega_{i} \omega_{i}\right.$ and $\left.S_{i j} S_{i j}\right)$ and each of their component fields (corresponding the each scale number, after filtering in curvelet domain) reach their maxima $\left(1 / k_{\text {peak }}\right)$, for the $1024^{3}$ case. Each wavelength has been non-dimensionalized with the average Kolmogorov scale $1 / k_{\text {peak }} \bar{\eta}$.
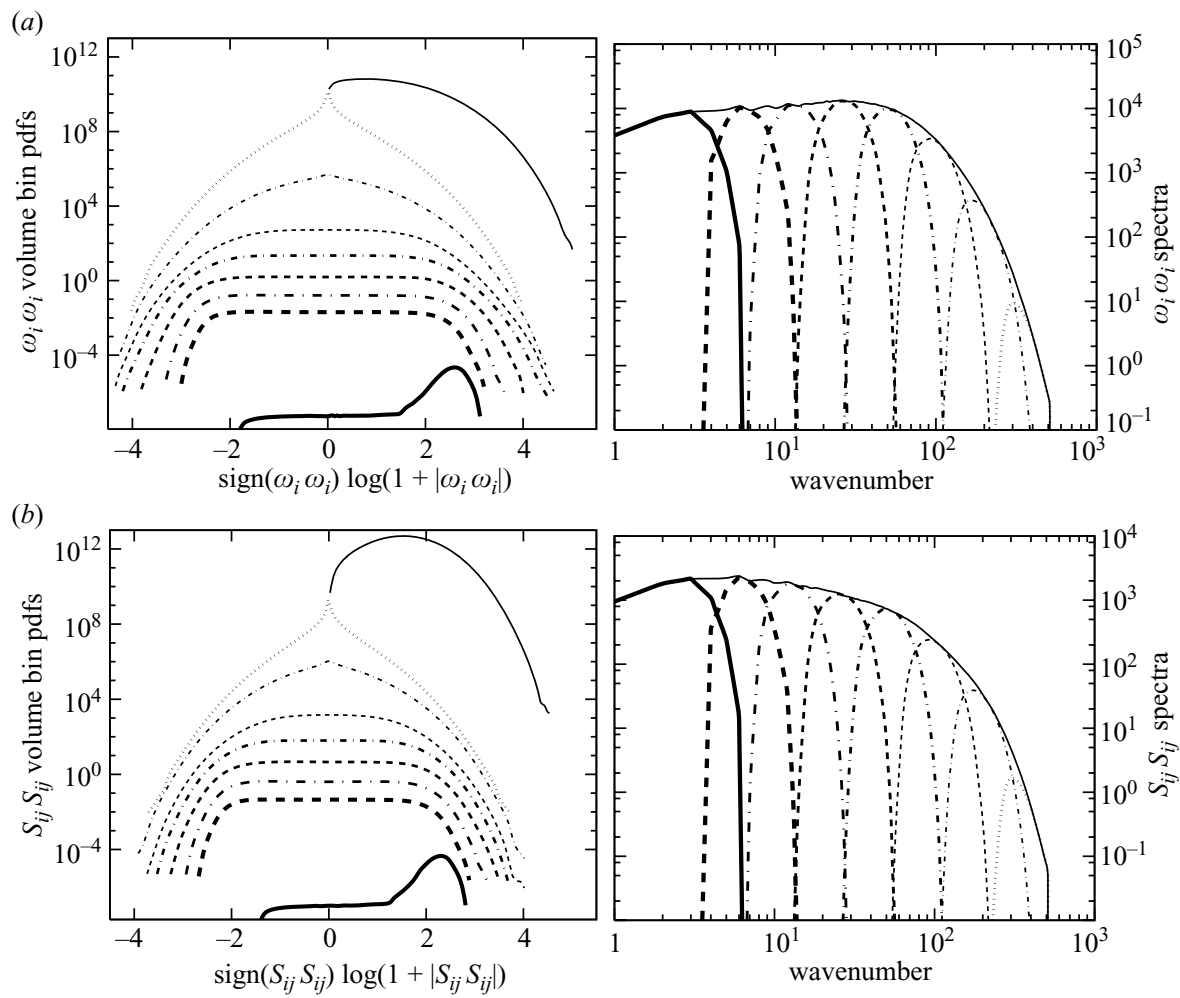

scales: all $\longrightarrow, 0 \longrightarrow, 1$

, 4 - . . . . . , 5

,6

FIGURE 4. Effect of the multi-scale decomposition in the $1024^{3}$ case for $\omega_{i} \omega_{i}(a)$ and $S_{i j} S_{i j}$ (b) fields on the volume pdfs in physical domain (left) and on the spectra in Fourier domain (right). Note that the volume pdfs have been shifted vertically to accommodate all scales and the original fields in a clearer view. Also, instead of using a log-scale in the abscissa of the pdf plots, since there are negative values for all filtered scales, a transformation of the form $\operatorname{sign}(x) \log (1+|x|)$ is used for each field $x$.

increases for increasing scale number (i.e. smaller scales), indicating that fluctuations of both fields are higher in the small scales, and therefore, that intermittency also increases for those smaller scales. Previous multi-scale studies of turbulence have shown this property (Kennedy \& Corrsin 1961; Meneveau 1991; Brasseur \& Wang 1992; Okamoto et al. 2007). Table 2 shows, for the original fields $\left(\omega_{i} \omega_{i}\right.$ and $\left.S_{i j} S_{i j}\right)$ containing all scales and for each individual scale, in the $1024^{3}$ case, the wavelength at 
(a)

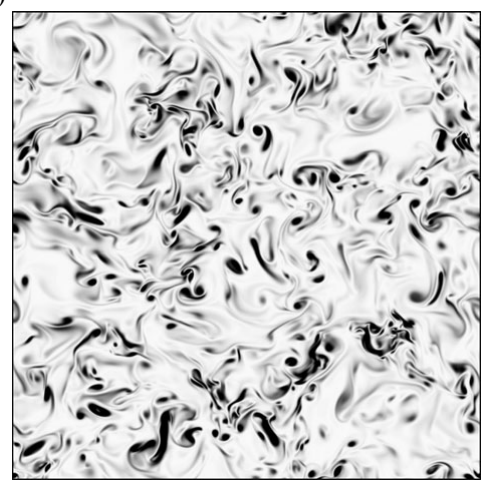

(b)

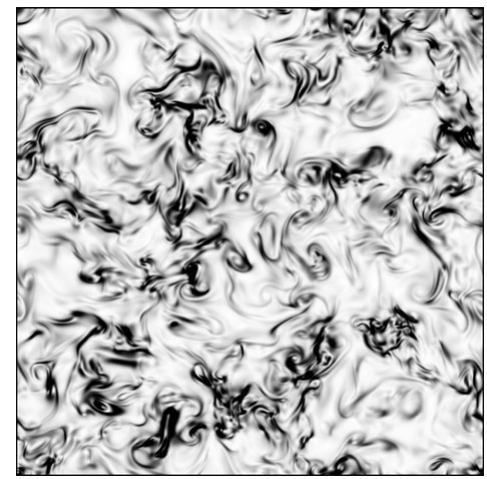

FIGURE 5. Plane cuts of $\omega_{i} \omega_{i}(a)$ and $S_{i j} S_{i j}(b)$ normal to one of the principal directions of the cubic domain at half its side length for the $1024^{3}$ case.


FiguRE 6. Zoomed parts of plane cuts of $\omega_{i} \omega_{i}(a)$ and $S_{i j} S_{i j}(b)$ in one of the principal directions of the cubic domain at half its side length for the three grid resolutions $256^{3}$ (left), $512^{3}$ (centre) and $1024^{3}$ (right). Greyscale has been renormalized to the zoomed region for better clarity.

which the corresponding spectrum reaches its maximum value, non-dimensionalized with the average Kolmogorov scale.

Figure 5 includes plane cuts of $\omega_{i} \omega_{i}(a)$ and $S_{i j} S_{i j}(b)$ fields, for the $1024^{3}$, at half the length of the physical domain in one of the principal directions of the cube. Zoomed parts of those plane cuts are shown in figure 6 for the three grid resolutions $\left(256^{3}, 512^{3}\right.$ and $1024^{3}$ from left to right) for both $\omega_{i} \omega_{i}(a)$ and $S_{i j} S_{i j}(b)$ fields. It can be observed that, particularly in the $256^{3}$ case, the smallest scales are quite different from the higher-resolution cases. Figure 7 shows zoomed parts of the plane cuts corresponding to the component field at scale number 5 for the three grid resolutions, which is the highest scale number attainable in the multi-scale decomposition of the $256^{3}$ case, and therefore, contains the structures at the smallest scales captured in this 

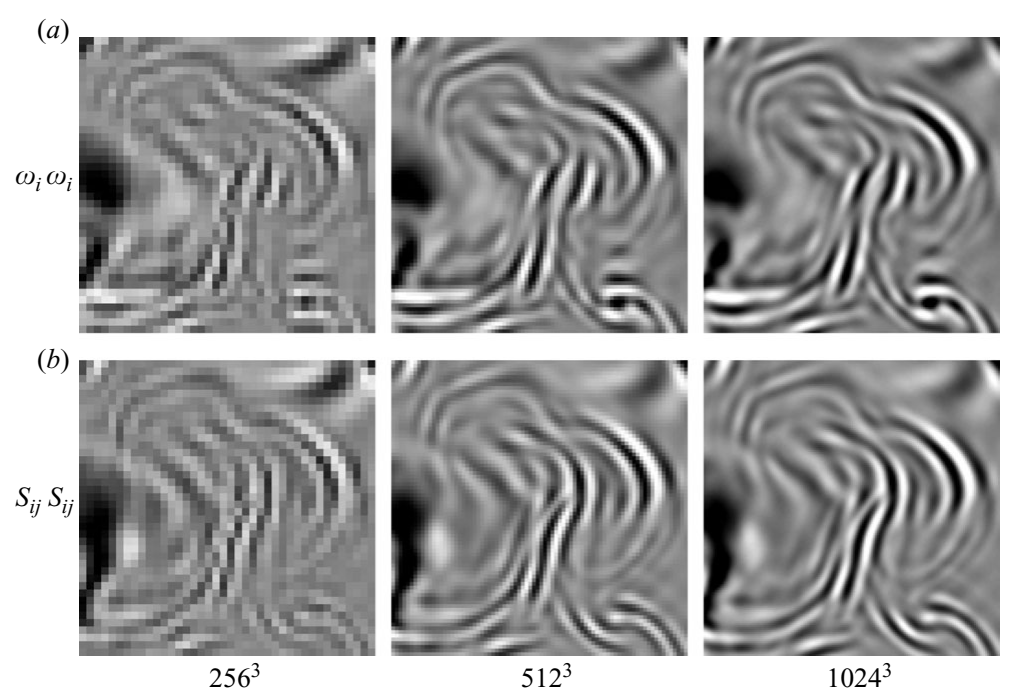

FIGURE 7. Zoomed parts of plane cuts of component field at scale number 5 for $\omega_{i} \omega_{i}$ (a) and $S_{i j} S_{i j}(b)$ in one of the principal directions of the cubic domain at half its side length for the three grid resolutions $256^{3}$ (left), $512^{3}$ (centre) and $1024^{3}$ (right). Greyscale has been renormalized to the zoomed region for better clarity.

flow at that grid resolution. It is clear from the two-dimensional fields that structures educed with the lowest grid resolution $256^{3}$ can be significantly different from the ones at higher grid resolutions, $512^{3}$ and $1024^{3}$. Intuitively, a geometrical characterization of those structures would be affected by that fact, and its effect would be noticed in the application of the methodology proposed above.

\subsection{Characterization and classification of individual structures}

In figure 8 , we present the three-dimensional visualization spaces (formed by $\hat{S}, \hat{C}, \lambda$ axes) with the glyphs (simple spheres in this case) representing each structure after its geometrical characterization. The top row corresponds to $\omega_{i} \omega_{i}$ and the bottom row to $S_{i j} S_{i j}$ for increasing grid resolution $\left(256^{3}, 512^{3}\right.$ and $\left.1024^{3}\right)$ from left to right. The spheres are scaled by the lognormalized area of the corresponding structure. The colour of each sphere represents the scale number to which it belongs. As in BP, the largest scale is not included in the analysis for being strongly dependent on the boundary and initial conditions. Neither is the smallest scale (for each grid resolution) to avoid interference between grid resolution effects and the iso-contouring process. The fact that we have three different grid resolutions for the equivalent field allows us to verify whether that interference occurs. This is discussed at the end of $\S 2.4$. Thus, scales $1-4,1-5$ and $1-6$ are represented for the $256^{3}, 512^{3}$ and $1024^{3}$ grid resolutions, respectively. Top views ( $\hat{S}-\hat{C}$ plane) of these visualization spaces are shown in figure 9 , where the differences between $\omega_{i} \omega_{i}$ and $S_{i j} S_{i j}$ fields can be better realized.

In figure 10, we show the breakdown by increasing scale number (top to bottom) of the three-dimensional visualization spaces for the $\omega_{i} \omega_{i}$ field for the three grid resolutions $\left(256^{3}, 512^{3}\right.$ and $\left.1024^{3}\right)$, increasing from left to right. Top views $(\hat{S}-\hat{C}$ plane) of each visualization space can be seen in figure 11. Figures 12 and 13 are the equivalent ones for $S_{i j} S_{i j}$. Figure 14 shows the number of structures analysed for each grid resolution and scale number (i.e. the number of glyphs represented in figures 10-13). 


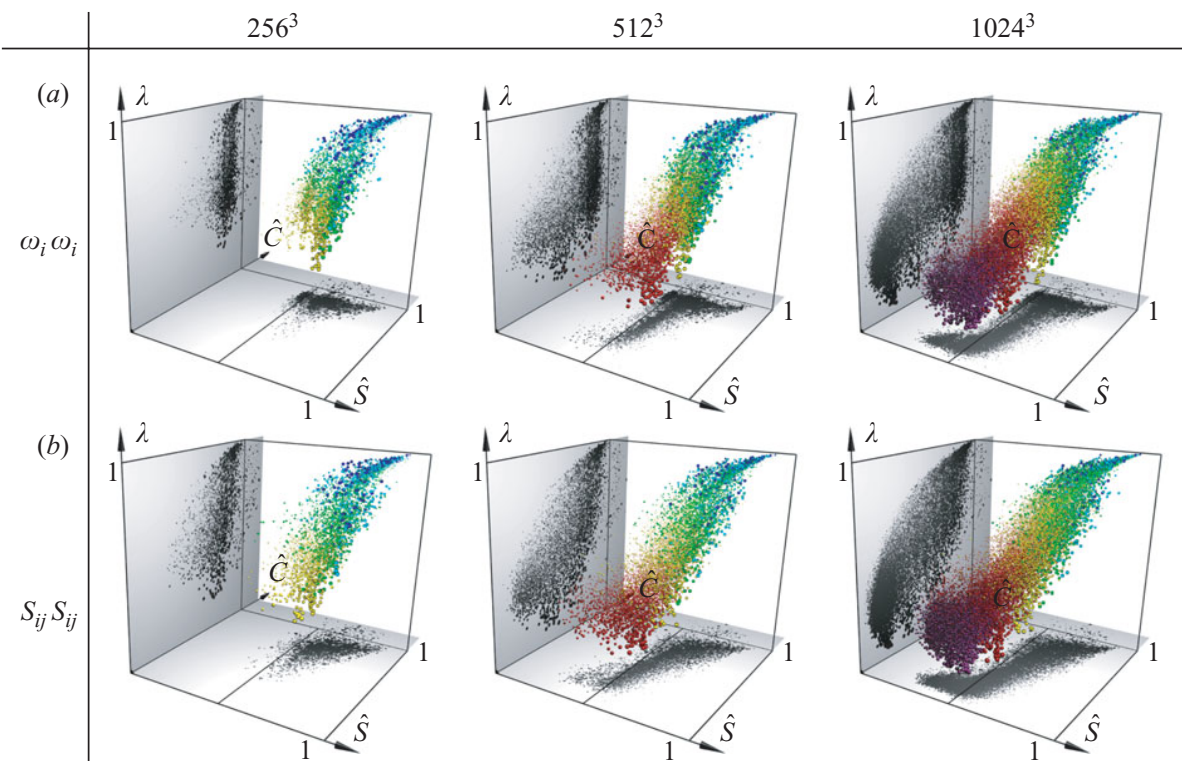

FIGURE 8. Three-dimensional views of the visualization spaces, with glyphs (spheres) representing educed structures, coloured by scale number - $\bullet$ (1), $\bullet$ (2), $\bullet$ (3), (4), $\bullet$ $(5), \bullet(6)$ - and scaled by the lognormalized area of the corresponding structure, for $\omega_{i} \omega_{i}(a)$ and $S_{i j} S_{i j}(b)$ at $256^{3}$ (left), $512^{3}$ (centre) and $1024^{3}$ (right) grid resolutions.

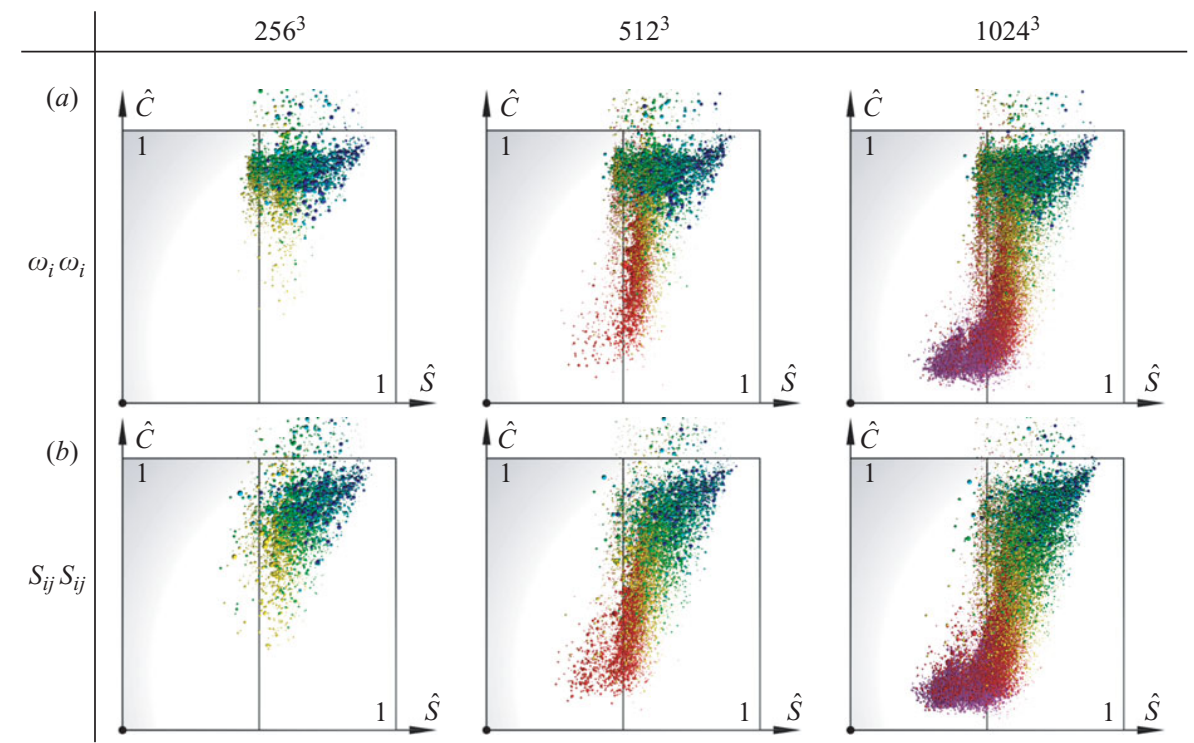

Figure 9. Top views ( $\hat{S}-\hat{C}$ plane) of the visualization spaces, with glyphs (spheres) representing educed structures, coloured by scale number - $\bullet(1), \bullet(2), \bullet(3), \bullet(4), \bullet(5), \bullet(6)-$ and scaled by the lognormalized area of the corresponding structure, for $\omega_{i} \omega_{i}(a)$ and $S_{i j} S_{i j}(b)$ at $256^{3}$ (left), $512^{3}$ (centre) and $1024^{3}$ (right) grid resolutions. 


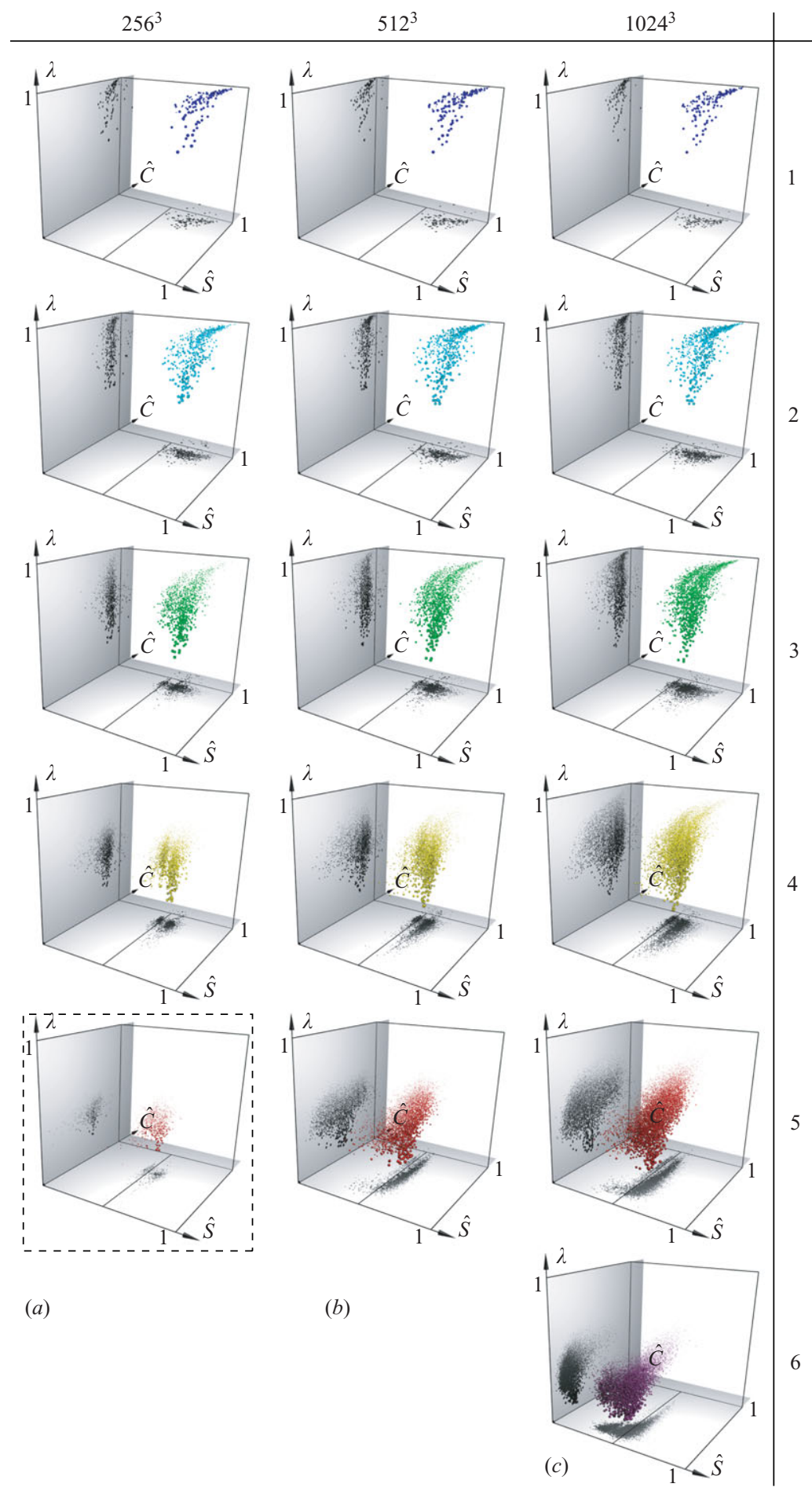

Figure 10. Breakdown, by scale number (increasing top to bottom), of three-dimensional views of the visualization spaces for $\omega_{i} \omega_{i}$ at $256^{3}(a), 512^{3}(b)$ and $1024^{3}(c)$ grid resolutions. 


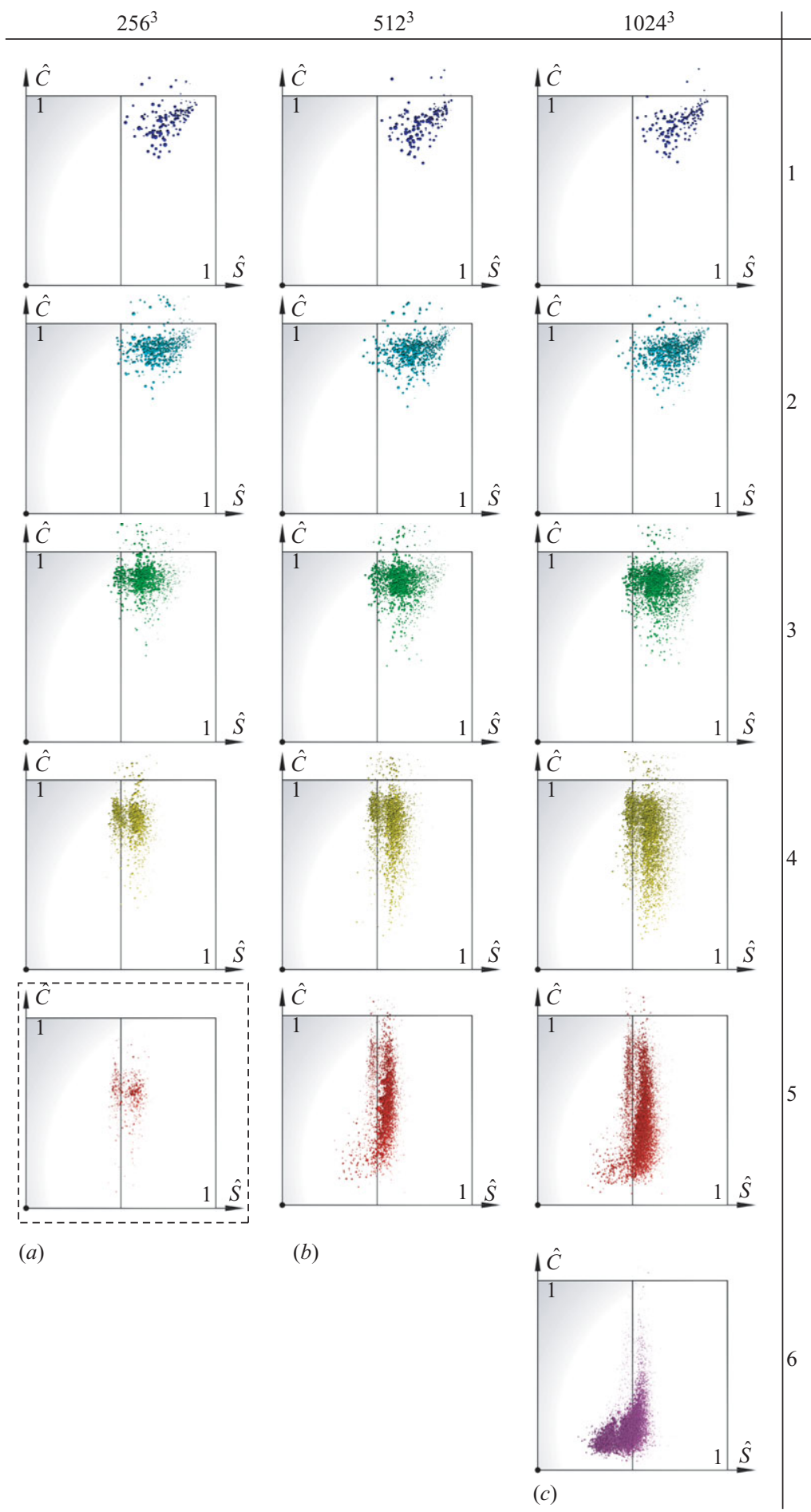

FIGURE 11. Breakdown, by scale number (increasing top to bottom), of top views $(\hat{S}-\hat{C}$ plane) of the visualization spaces for $\omega_{i} \omega_{i}$ at $256^{3}(a), 512^{3}(b)$ and $1024^{3}(c)$ grid resolutions. 


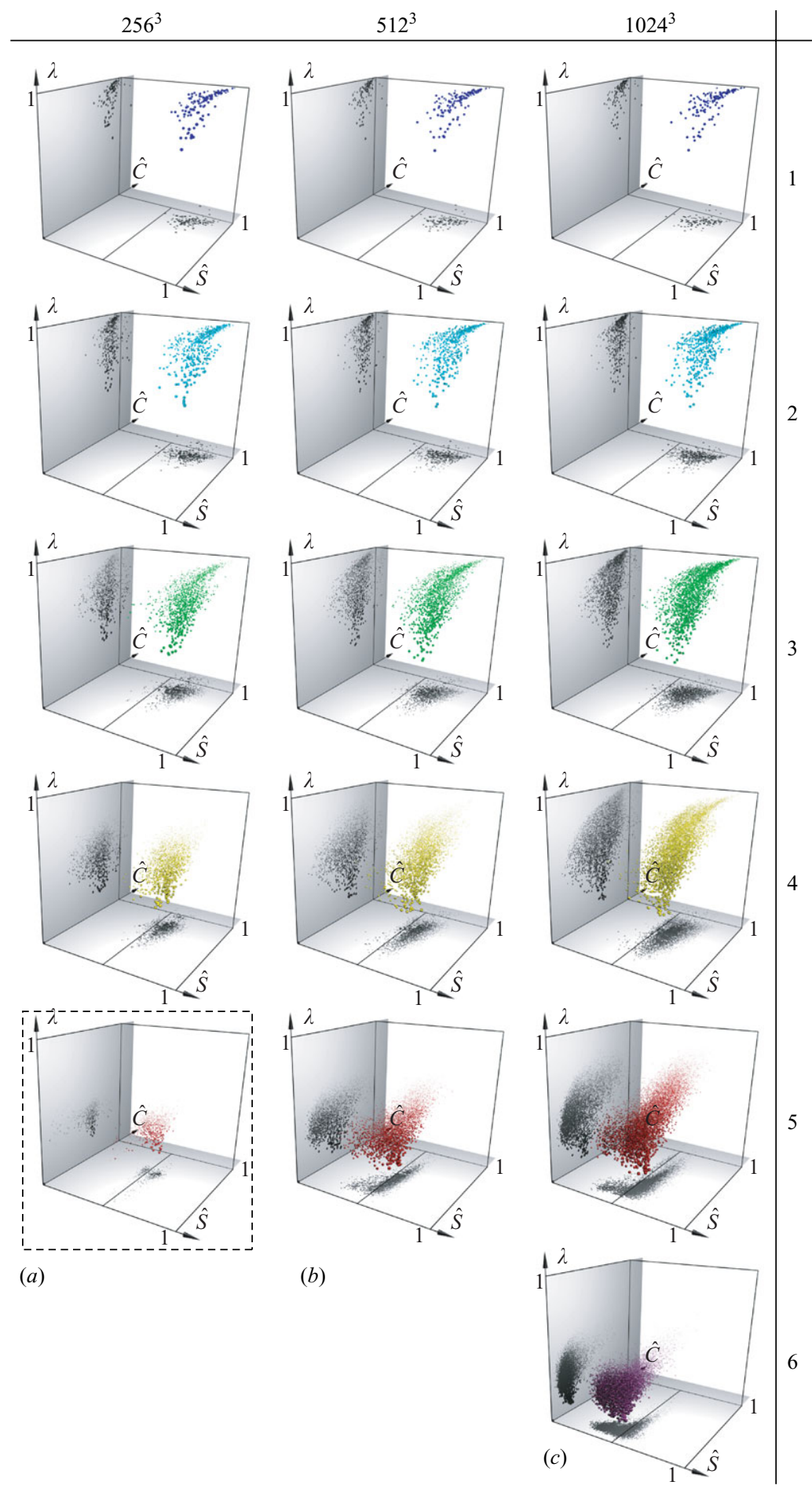

FIGURE 12. Breakdown, by scale number (increasing top to bottom), of three-dimensional views of the visualization spaces for $S_{i j} S_{i j}$ at $256^{3}(a), 512^{3}(b)$ and $1024^{3}(c)$ grid resolutions. 


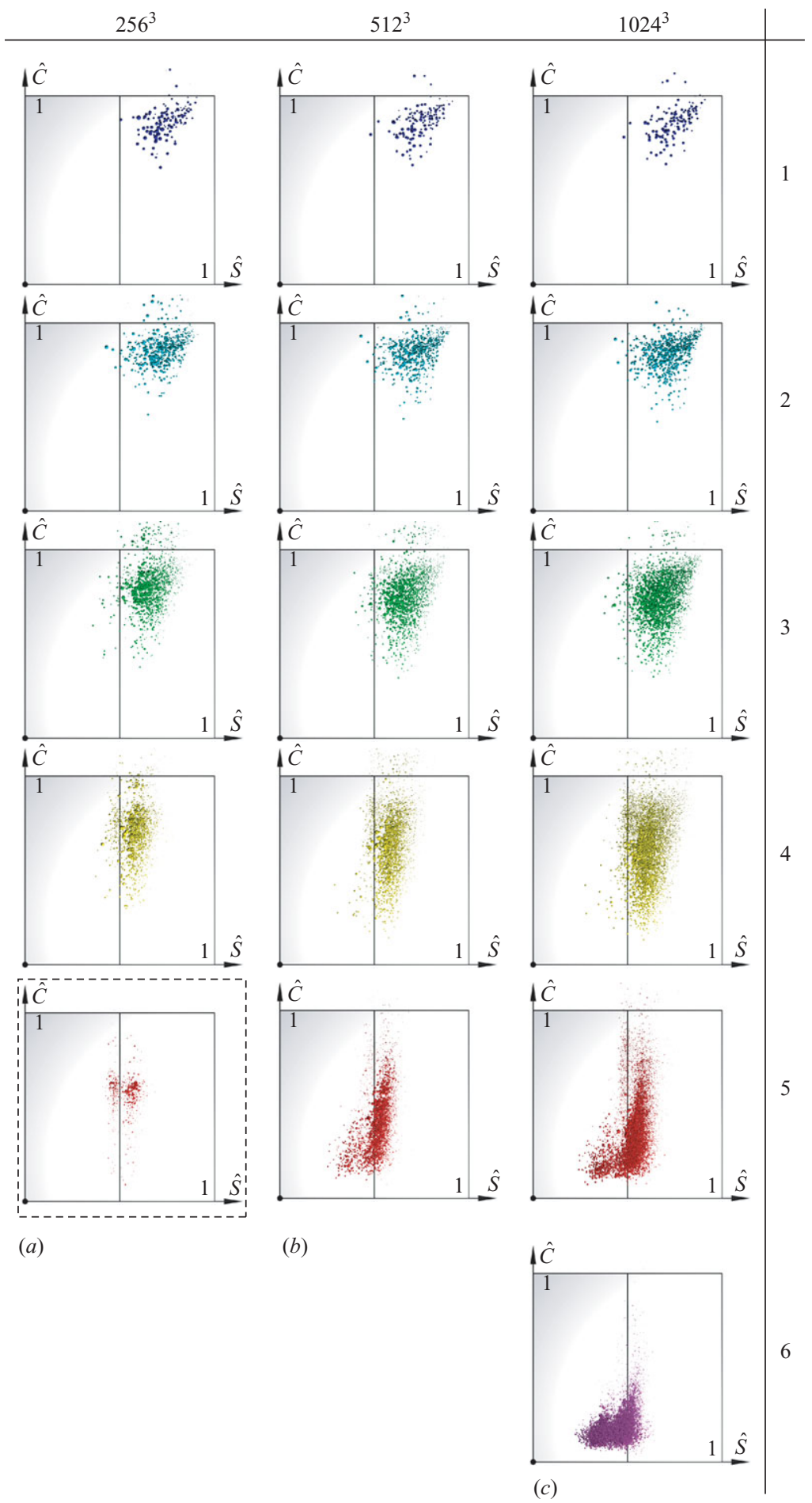

FIGURE 13. Breakdown, by scale number (increasing top to bottom), of top views $(\hat{S}-\hat{C}$ plane) of the visualization spaces for $S_{i j} S_{i j}$ at $256^{3}(a), 512^{3}(b)$ and $1024^{3}(c)$ grid resolutions. 


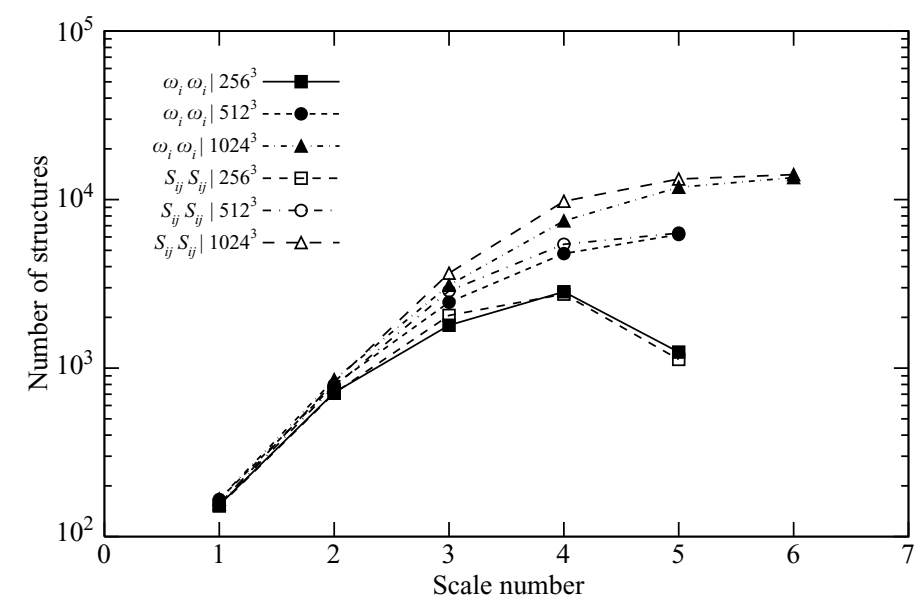

FIGURE 14. Number of structures analysed for each grid resolution and scale number.

We first discuss the case with the highest resolution $\left(1024^{3}\right)$. Structures of both $\omega_{i} \omega_{i}$ and $S_{i j} S_{i j}$ fields show a continuous transition of their corresponding glyphs in the visualization space, with varying scale (see right plots of figures 8 and 9). Structures at the largest scale of both fields (top right corner of figures 10 and 12) are mainly blob-like; some get closer to the tube-like region with small stretching (high $\lambda$ ). At the smallest scale (bottom right corner of figures 10 and 12), dominant structures of both fields are sheet-like (low values of $\hat{C}$ and $\lambda$ ). The intermediate scales present a different behaviour for each field: $\omega_{i} \omega_{i}$ shows a high concentration of structures near the tube-like region (see scale numbers 3 and 4 in figure 11), highly stretched particularly for the smaller scales; the transition to sheet-like structures appears to be significant at scale number 4 and becomes obvious at scale number 5 , for which dominant structures span across almost all values of $\hat{C}$. On the other hand, $S_{i j} S_{i j}$ structures concentrate less in the tube-like region (see figure 9 and compare scale numbers 3 and 4 of $\omega_{i} \omega_{i}$ in figure 11 with those of $S_{i j} S_{i j}$ in figure 13), while they show, at all intermediate scales, many more structures with smaller values of $\hat{C}$, characteristic of sheet-like geometries. The transition to sheet-like structures begins earlier, at scale number 3 , for $S_{i j} S_{i j}$ than for $\omega_{i} \omega_{i}$, and is completed by scale number 5 .

\subsection{Effect of grid resolution in the geometry of structures}

From figures 8 and 9 , it is observed that the $256^{3}$ case does not capture well the dominance of sheet-like structures that occurs in both $\omega_{i} \omega_{i}$ and $S_{i j} S_{i j}$ in the small scales (scales numbers from 4 onwards). Figures 12 and 13 show, for $S_{i j} S_{i j}$, a tendency towards sheet-like structures at the smallest scale studied for the $256^{3}$ case, although the smaller values of $\hat{C}$ present in the $512^{3}$ and $1024^{3}$ for the same scale number are not captured in the $256^{3}$ case either. This is even more pronounced in the $\omega_{i} \omega_{i}$ field (see, in particular, figure 11), for which the departure from tube-like towards the sheet-like structures present at higher grid resolutions is not obvious at all in the $256^{3}$ case.

The $512^{3}$ case performs better than the $256^{3}$ case in describing the geometry of the structures at the scales of study, when each one is compared with its immediately higher grid resolution. For example, the visualization space at scale number 5, the smallest scale analysed for $512^{3}$, is rather similar to the $1024^{3}$ case (columns $(b)$ and 
(c) of figures 10-13), where structures with geometries transitioning from the tubelike region to the strongly sheet-like region are captured at both resolutions in more similar proportions. The $1024^{3}$ case still shows a higher concentration of sheet-like structures, particularly for the $S_{i j} S_{i j}$ case.

These results are consistent with the observations of HF. They identified multiple modes of the stretched spiral vortex (Lundgren 1982) in the numerical database and investigated their formation processes. They found that the highest grid resolution $k_{\max } \bar{\eta} \approx 4$ was needed to eliminate the fragmentation of sheets for a precise capture of the spiral turns (sheet-like) of those structures and for a proper study of the dissipation field. Schumacher, Sreenivasan \& Yeung (2005) also found the necessity of resolving sub-Kolmogorov scales when studying the very fine structures in scalar mixing, where sheets are also dominant in the scalar dissipation field. Sreenivasan (2004) proposed a revised grid resolution criterion, based on intermittency arguments, which uses estimates of the ratio of maximum to average dissipation obtained from measured multi-fractal exponents (see Sreenivasan \& Meneveau 1988) much more stringent than the traditional $k_{\max } \bar{\eta} \approx 1$ criterion. As previously noted, the largest and smallest scales for each grid resolution were omitted. The largest scale is dependent on boundary and initial conditions applied and, therefore, is of less interest in this particular study. The smallest scale was not analysed to avoid interference with the iso-contouring step due to grid resolution effects. But three grid resolutions of the same flow realization provide the opportunity to verify whether that last statement holds. For that reason, figures 10-13 include, for the $256^{3}$ case (column $(a)$ ), one additional visualization space corresponding to scale number 5 (framed in a dashedline box). When compared to the homologous scale number for the higher grid resolutions, it can be seen that the strong sheet-like character of the structures is not well captured in the $256^{3}$. A possible explanation is that sheet-like structures at that resolution are more fragmented into smaller structures (part of the original ones). Some will still be sheet-like but their tube-like area coverage increases since the nearly planar area is reduced and the surface is still closed, which results in higher values of $\hat{C}$ directly affecting their location in the visualization space. Some others can even result in small blob-like structures or, in general, rather distinct geometries than the original sheets of which they are fragments. This is confirmed when the population of individual structures at that scale number is evaluated, since there is a high increase of small-area structures. The pixelization effect seen in figure 7 for the two-dimensional plane cuts, when extended to three-dimensions (where the iso-contours, and thus the individual structures, are obtained) can help to visualize the scenario described above.

\subsection{Clustering results for the $1024^{3}$ case}

As part of the classification step of the methodology, clustering techniques are applied to the structures obtained from all merged scales under study. This is done independently for structures of $\omega_{i} \omega_{i}$ and $S_{i j} S_{i j}$. Only the $1024^{3}$ database is considered here. The number of structures present at each scale largely increases with the scale number (i.e. for smaller and smaller scales). Therefore, geometries of structures of larger scales could be under-represented in the clustering process. To avoid that situation, a stratified random sampling with a disproportionate allocation, based on the standard deviation of the population of each scale number, is applied among the present scales prior to the clustering algorithm. See Appendix B for more details.

The results of the clustering algorithm applied individually to $\omega_{i} \omega_{i}$ and $S_{i j} S_{i j}$ structures are presented in figure 15. An optimum number of three clusters was automatically obtained by the algorithm for $\omega_{i} \omega_{i}$, while the structures of $S_{i j} S_{i j}$ were 
(a)
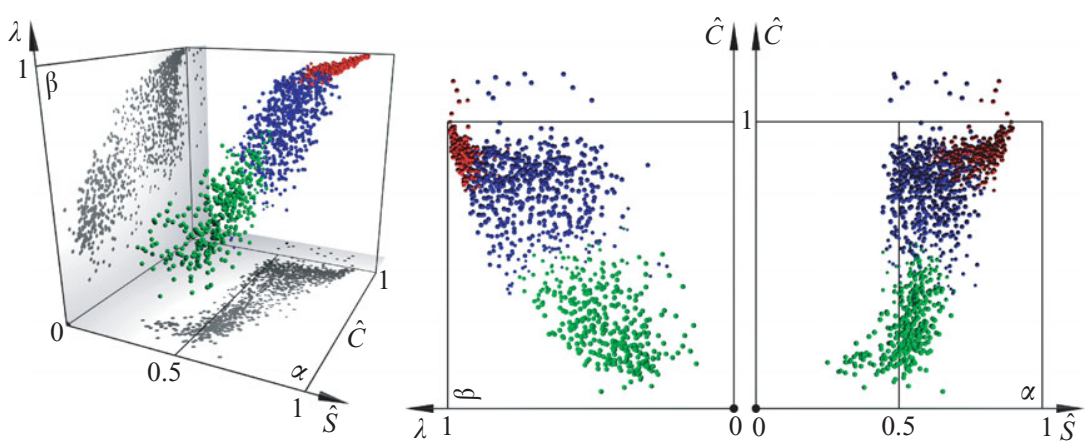

(b)
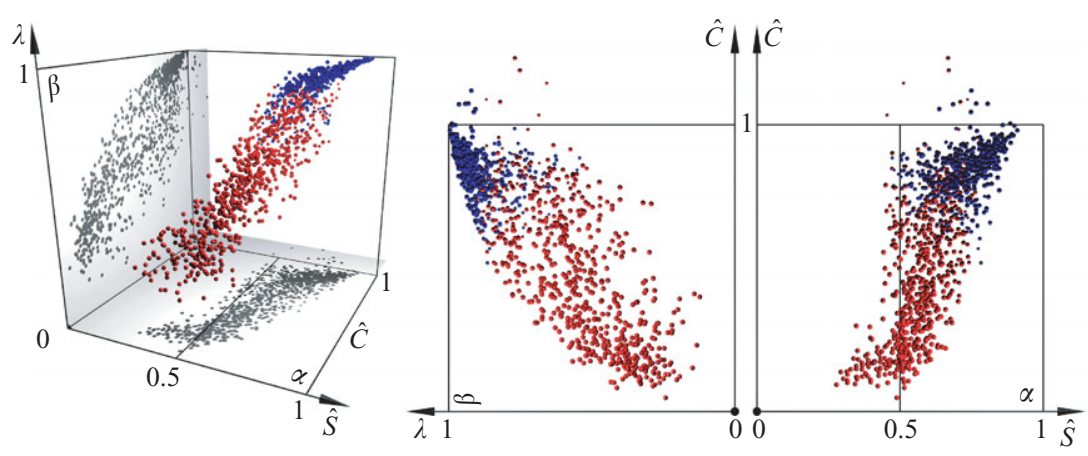

FIGURE 15. Clustering results in the visualization space - three-dimensional view (left) and lateral (centre) and top (right) projections - with glyphs (spheres) representing the optimum clusters of structures educed from the stratified random sample with optimum allocation of the sets of $\omega_{i} \omega_{i}(a)$ and $S_{i j} S_{i j}(b)$ structures. Glyphs are scaled by the normalized silhouette value, which indicates the degree of membership of that element to the assigned cluster.
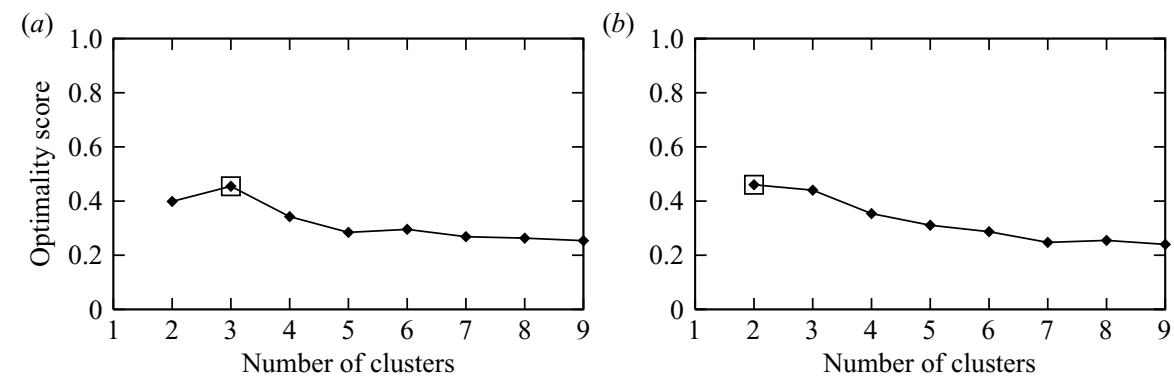

FIGURE 16. Optimality scores for different number of clusters obtained during the application of the clustering algorithm to the set of structures of $\omega_{i} \omega_{i}(a)$ and $S_{i j} S_{i j}(b)$ independently. Optimum number of clusters (square point) of 3 and 2 were automatically determined for $\omega_{i} \omega_{i}$ and $S_{i j} S_{i j}$, respectively.

optimally clustered in two groups. Optimality scores obtained during the automatic determination of the number of clusters for $\omega_{i} \omega_{i}(a)$ and $S_{i j} S_{i j}(b)$ are plotted in figure 16. The optimality score is computed as the mean value of the silhouette coefficient of all the clustered elements minus the standard deviation of those silhouette coefficients. Higher mean values of the silhouette coefficient imply that the elements were clustered in groups where they have a high degree of membership. Small values 
of the standard deviation of the silhouette values indicate homogeneity in that level of membership among the clustered elements. The combination of a high mean and a low standard deviation is sought for an optimum clustering result. We note that the optimality scores obtained are rather low (less than $0.5,1$ being the maximum achievable), even for the optimum number of clusters. Also, the optimality score associated with the optimum number of clusters determined for each case does not differ significantly from the rest. These two facts indicate that the elements to cluster are organized as a cloud of points continuously distributed throughout the feature space of parameters used for clustering, instead of being organized in well-distinguishable groups that would result in higher optimality scores and more variation among those scores for non-optimal number of clusters. A projection of that feature space is the visualization space where the results have been plotted, where it is also possible to see the continuously distributed cloud of glyphs.

\subsection{Discussion}

The dominance of tube-like structures at intermediate scales of $\omega_{i} \omega_{i}$ is consistent with the presence of so-called 'worms' reported in the fluid mechanics literature (see, for example, Siggia 1981; Jiménez et al. 1993). Tube-like structures also appear at intermediate scales of $S_{i j} S_{i j}$ but in less proportion than for $\omega_{i} \omega_{i}$. At all scales analysed, $S_{i j} S_{i j}$ shows, on average, more planar geometries than $\omega_{i} \omega_{i}$. Also, the transition to sheet-like structures occurs earlier (larger scale) for $S_{i j} S_{i j}$.

The maximum departure between the spectra of $\omega_{i} \omega_{i}$ and $S_{i j} S_{i j}$ occurs at the intermediate scales (scale numbers 3 and 4 ), as observed in figure 4 . This seems to translate into differences in the geometrical character of structures of $\omega_{i} \omega_{i}$ and $S_{i j} S_{i j}$ at those scales numbers. In physical space, the higher concentration of tube-like structures found in the enstrophy field might be one geometrical link to the higher intermittency of this field, when compared to the dissipation field.

At the smallest scale, both fields show a clear dominance of sheet-like structures. They appear highly stretched, that is, with small thickness, but their spatial extent can be significant. Instabilities of vortex sheets have been suggested as a primary mechanism responsible for the generation of vortex tubes in turbulent flows. Vincent \& Meneguzzi (1994) found that the production of vortex sheets and their subsequent roll-up, forming tubes, shows a strong correlation between scales and occurs in a onestep process (in contrast with Richardson multi-step cascade picture). Furthermore, they explain the alignment of vorticity with the intermediate strain-rate eigenvector as a consequence of vorticity sheet production by strong strain, instead of tube formation. HF identified the stretched spiral vortex (Lundgren 1982) in homogeneous isotropic turbulence, appearing in three modes (two symmetric and one antisymmetric), that involve one or multiple vortex sheets interacting to generate tubes. While the stretched spiral vortex was not found in the work of Vincent \& Meneguzzi (1994), that was attributed by HF to the increased grid resolution required to avoid fragmentation of the spiral turns. The correlation of geometries for $\omega_{i} \omega_{i}$ and $S_{i j} S_{i j}$ at the smallest scale is consistent with the known feature of sheets, in which both strain and rotation rates are large and correlated (Ruetsch \& Maxey 1992; Horiuti \& Takagi 2005)

Previous studies of the enstrophy field also suggest (see Nomura \& Post 1998, and the references therein) that its geometry depends on its local magnitude: intense regions appear tube-like, while moderate enstrophy regions seem to be more sheetlike. These considerations are, nevertheless, independent of the scale. Besides the iso-contour value of the mean plus twice the standard deviation of each filtered component field, whose results have been presented here, we also examined the 
mean plus three times the standard deviation, with no significant differences of the geometries educed for each field. A wider range of iso-contour values would help clarify the sensitivity of the geometries. Nonetheless, we note that the categorization of globally intense or moderate values of the original field (containing all scales) based on the iso-contour value is not directly applicable to its component fields after the multi-scale decomposition.

The $256^{3}$ grid resolution $\left(k_{\max } \bar{\eta} \approx 1\right)$ was unable to reproduce the predominance of highly stretched sheet-like structures found for the smaller scales at higher grid resolutions. This indicates that sub-Kolmogorov scales must be resolved for a proper geometrical study of the smallest structures in turbulence, as has been suggested in the literature (see Schumacher et al. 2005; Horiuti \& Fujisawa 2008) when studying intermittent fields: their high fluctuations (manifested in the long tails of the volume pdfs in physical space) will occur at very fine scales. These, in general, can be substantially smaller than the average Kolmogorov length-scale, $\bar{\eta}=\left(v^{3} /\langle\epsilon\rangle\right)^{1 / 4}$, defined in terms of the average rate of kinetic energy dissipation per unit mass, $\langle\epsilon\rangle$, and the viscosity of the fluid, $v$, and traditionally used to define the largest dynamically significant wavenumber resolved in DNS, $k_{\max }$, such that $k_{\max } \bar{\eta} \approx 1$. As noted in $\S 2.2$, the volume pdfs of the different scale component fields obtained from $\omega_{i} \omega_{i}$ and $S_{i j} S_{i j}$ show wider ranges for smaller scales, indicating that higher fluctuations of those two fields occur in the small scales and confirming the intermittency of both fields.

The initial condition of the DNS used in the present study was given according to (2.1) in HF. Since $E(k) \propto \exp \left\{2\left(\frac{k}{k_{p}}\right)^{2}\right\}$, and $k_{p}=2$, at an early stage of the temporal development of the flow field, a large-scale blob or an extensive sheet-like vortical structure is formed. Although this paper deals with the DNS data at a later stage in which the enstrophy is maximum, application of this methodology at an early stage may exhibit predominance of structures in the blob region of the visualization space. We can hypothesize that by straining and stretching of these blobs, they evolve into thin sheets. As was shown in HF, these stretched sheets often form mode 3 configuration. Then, they are converted into mode 1 or 2 configuration. In addition, these stretched sheets roll up, and numerous mode 1 spiral vortices are created. Visualization study indicates that many mode 3 spiral vortices are discernible at the earlier stages, but mode 1 (or 2) vortices become dominant at the later stages (see figure 24 in $\mathrm{HF}$ for a schematic).

\section{Assessment of the new non-local methodology complementing existing local methods}

In this section, the non-local methodology for the study of the geometry of structures in turbulence introduced in BP and used in $\S 2$ is utilized to complement two local criteria present in the literature for the identification of vortex tubes and vortex sheets in turbulent flows. The purpose is to provide a qualitative and quantitative assessment of the geometrical aspects of those local identification criteria. This is performed by applying the non-local methodology to confirm whether the educed structures show the expected geometries. The local and non-local methods are applied to the same numerical database referred to in $\S 2$.

\subsection{Local identification criteria}

Among the various local criteria found in the literature, here we consider those used in Horiuti \& Takagi (2005) and HF for educing vortex tubes and sheets, which are outlined below. A point is considered to belong to a vortex tube core where the 
second invariant, $Q$, of the velocity-gradient tensor, $\partial u_{i} / \partial x_{j}$, has a sufficiently large value. $Q$ is defined as

$$
Q \equiv \frac{1}{2}\left[\left(\frac{\partial u_{i}}{\partial x_{i}}\right)^{2}-\frac{\partial u_{i}}{\partial x_{j}} \frac{\partial u_{j}}{\partial x_{i}}\right]
$$

For incompressible flow, $\partial u_{i} / \partial x_{i}=0$, and $Q$ is related to $\omega_{i} \omega_{i}$ and $S_{i j} S_{i j}$ by

$$
2 Q=-\frac{\partial u_{i}}{\partial x_{j}} \frac{\partial u_{j}}{\partial x_{i}}=\Omega_{i j} \Omega_{i j}-S_{i j} S_{i j}=\frac{1}{2} \omega_{i} \omega_{i}-S_{i j} S_{i j} .
$$

The condition $Q>0$ was first used by Hunt et al. (1988), in combination with the additional constraint of the pressure being lower than ambient, to define vortex tubes. The Poisson's equation for pressure, $p$, in incompressible turbulent flow (see Bradshaw \& Koh 1981) can be rewritten as

$$
\frac{1}{\rho} \frac{\partial^{2} p}{\partial x_{i} \partial x_{i}}=-\frac{\partial u_{i}}{\partial x_{j}} \frac{\partial u_{j}}{\partial x_{i}}=2 Q=\frac{1}{2} \omega_{i} \omega_{i}-S_{i j} S_{i j} .
$$

Therefore, $Q$ is a pressure source term. Also, from the latter equality, enstrophy acts as a source term, while dissipation acts as a sink term of pressure. There are some situations in which the $Q$-criterion is not adequate for educing vortex tubes, as shown in Jeong \& Hussain (1995) (e.g. conically symmetric vortex, axisymmetric axial vortex within a vortex ring, Bödewadt vortex).

For educing vortex sheets, the method proposed by Horiuti \& Takagi (2005) is used in this section. At any given point, the eigenvalues of the symmetric second-order tensor $A_{i j} \equiv S_{i k} \Omega_{k j}+S_{j k} \Omega_{k i}$ are reordered as $\left[A_{i j}\right]_{\omega},\left[A_{i j}\right]_{+}$and $\left[A_{i j}\right]_{-}$. Here, $\left[A_{i j}\right]_{\omega}$ is the eigenvalue whose corresponding eigenvector is most aligned with the vorticity field, $\omega_{i}$, at that point. $\left[A_{i j}\right]_{+}$and $\left[A_{i j}\right]_{-}$are the remaining largest and smallest eigenvalues (in an algebraic sense), respectively. The eigenvalues, $\vartheta$, of $A_{i j}$ can be obtained from the depressed cubic equation

$$
\vartheta^{3}-\frac{1}{2} A_{i j} A_{j i} \vartheta+\frac{1}{3} A_{i j} A_{j k} A_{k i}=0 .
$$

Note that there is no term in $\vartheta^{2}$ for being $A_{i i}=0$, due to the symmetry of $S_{i j}$ and antisymmetry of $\Omega_{i j}$. Iso-contours of $\left[A_{i j}\right]_{+}$are considered vortex sheets. This method takes advantage of the known feature of vortex sheets, in which both strain rate and vorticity are large and correlated, reflected in $\left[A_{i j}\right]_{+}$. Horiuti \& Takagi (2005) explain the advantages of this identification criterion over previously existing ones also based on that feature of vortex sheets (see, for example, Tanaka \& Kida 1993).

\subsection{Application of non-local methodology}

Once the sets of iso-surfaces of $Q$ and $\left[A_{i j}\right]_{+}$are obtained, the non-local methodology introduced in BP is applied to both sets. The multi-scale decomposition is not used in the extraction step, since the purpose is to assess the geometrical character of the iso-surfaces extracted by the local criteria. A multi-scale decomposition of the $Q$ and $\left[A_{i j}\right]_{+}$scalar fields could be applied beforehand (as was done for $\omega_{i} \omega_{i}$ and $S_{i j} S_{i j}$ in $\S 2$ ), and then iso-contours of the component fields could be independently obtained, but the meaning of the educed structures would not be the same as those obtained by iso-contouring the original fields of $Q$ and $\left[A_{i j}\right]_{+}$, and the purpose of the assessment of the local criteria would be lost. While the three grid resolutions are also available for $Q$ and $\left[A_{i j}\right]_{+}$fields, only the finest (i.e. $1024^{3}$ ) is used, since this section is not intended to evaluate the effect of the grid resolution in $Q$ and $\left[A_{i j}\right]_{+}$structures. 



FIGURE 17. Volume pdfs in physical domain $(a)$ and spectra in Fourier domain $(b)$ of $Q$ and $\left[A_{i j}\right]_{+}$fields for the $1024^{3}$ grid resolution. Note that the volume pdfs use a transformation of the form $\operatorname{sign}(x) \log (1+|x|)$ in the abscissa coordinate, and that curves for $Q$ and $\left[A_{i j}\right]_{+}$fields use two different vertical axes (both in the pdfs and the spectra), shifted one decade for a clear view (non-intersecting curves).

(a)

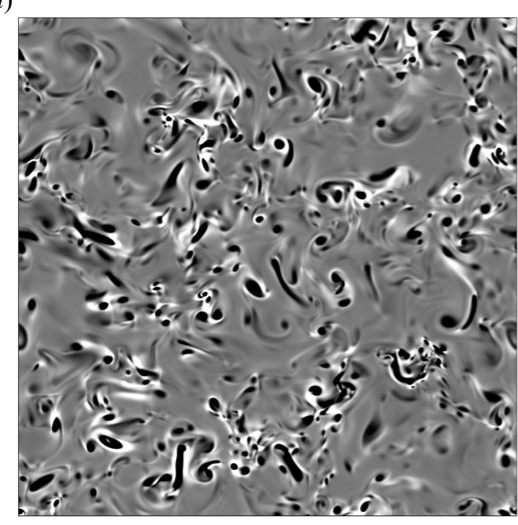

(b)

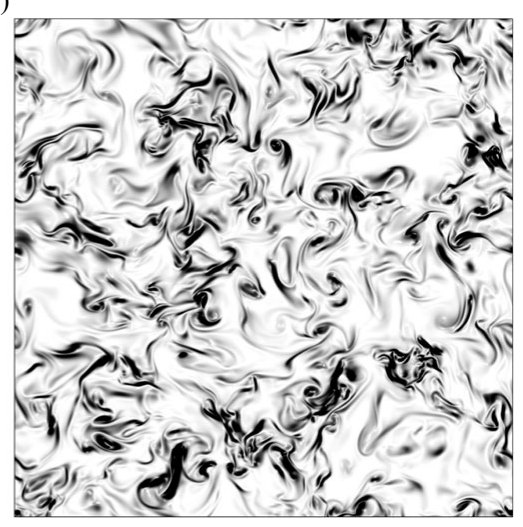

FIGURE 18. Plane cuts of $Q(a)$ and $\left[A_{i j}\right]_{+}(b)$ fields normal one of the principal directions of the cubic domain, at half its side length.

Figure 17 shows plots of the volume pdfs $(a)$ and the spectra $(b)$, in Fourier space, of the two fields, $Q$ and $\left[A_{i j}\right]_{+}$, for the finest grid resolution $\left(1024^{3}\right)$. The two plane cuts in figure 18 correspond to $Q(a)$ and $\left[A_{i j}\right]_{+}(b)$ fields, and were obtained normally to one of the principal directions of the cubic domain at half the side length. Iso-surfaces of $Q$ and $\left[A_{i j}\right]_{+}$extracted at contour values equal to the mean plus 5 and 4 times, respectively, the standard deviation of each field are presented in figure 19. These contour values are approximately the same ones used in HF (1200 for $Q$ and 1000 for $\left.\left[A_{i j}\right]_{+}\right)$to educe vortex tubes and sheets. The visualization spaces in figure 20 contain glyphs corresponding to the geometrical characterization of the individual structures shown in figure 19, with the same colouring scheme (blue used for $Q$ structures and red for $\left[A_{i j}\right]_{+}$structures). It is observed that structures of $Q$ tend to be located near the $(\hat{S}, \hat{C})=(1 / 2,1)$ region, where tube-like structures are generally located, and present moderate-to-high stretching. On the contrary, $\left[A_{i j}\right]_{+}$structures appear much closer to the $\hat{C}=0$ region, thus corresponding to more planar geometries (sheet-like), and with lower values of $\lambda$, implying more stretched structures. 
(a)

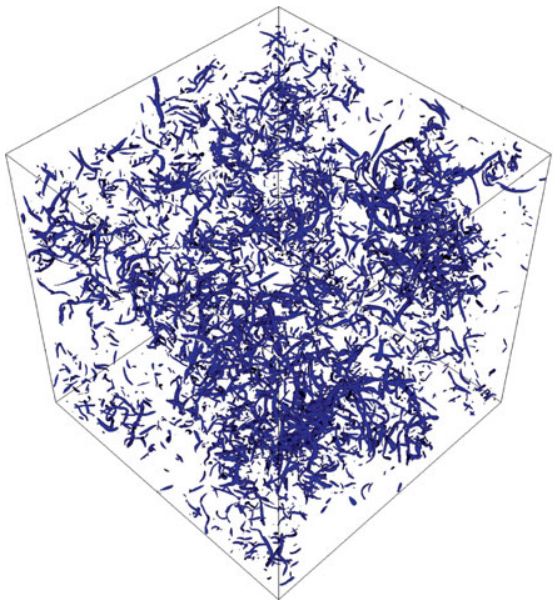

(b)



FIGURE 19. Iso-contours of $Q(a)$ and $\left[A_{i j}\right]_{+}(b)$ fields extracted at their mean plus 5 and 4 times their standard deviation, respectively.
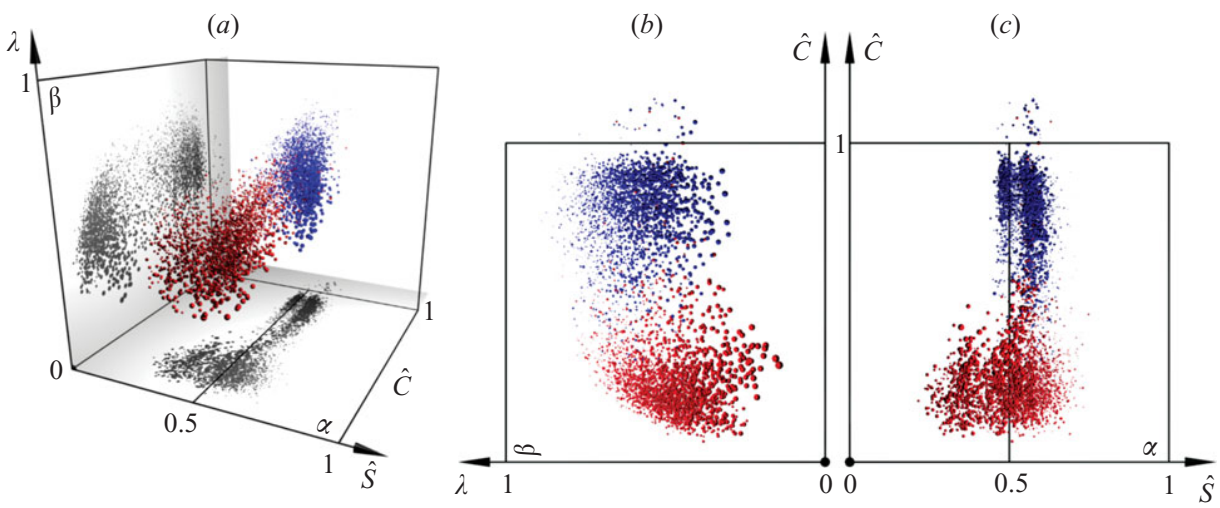

FIGURE 20. Visualization space - three-dimensional view $(a)$ and lateral $(b)$ and top (c) projections - with glyphs (spheres) representing educed structures of $Q$ (blue) and $\left[A_{i j}\right]_{+}$ (red), scaled by the lognormalized area of each corresponding structure.

The clustering algorithm described in the classification step of the non-local methodology is applied to the set of structures formed by the union of both sets of iso-surfaces of $Q$ and $\left[A_{i j}\right]_{+}$fields, without any a priori distinction of structures of those two sets. This means that the algorithm has no knowledge of whether individual structures were extracted from $Q$ or $\left[A_{i j}\right]_{+}$fields. Owing to the equivalent number of structures educed for $Q$ and $\left[A_{i j}\right]_{+}$and the similar standard deviations of each population, the pre-clustering stratified random sampling with disproportionate allocation in this case results in practically the union of the complete sets. Then, structures are clustered based solely on their geometrical characterization given by set of parameters $\left\{\hat{S}, \hat{C}, \lambda, d_{u}^{S}, d_{l}^{S}, d_{u}^{C}, d_{l}^{C}\right\}$ that define the feature space outlined in $\S 2$ and described in BP. An optimum number of clusters of 2 was found (see figure 22), and the resulting clusters are shown in figure 21, where each colour corresponds to a different cluster. Glyphs (spheres) in that figure are scaled by the normalized 

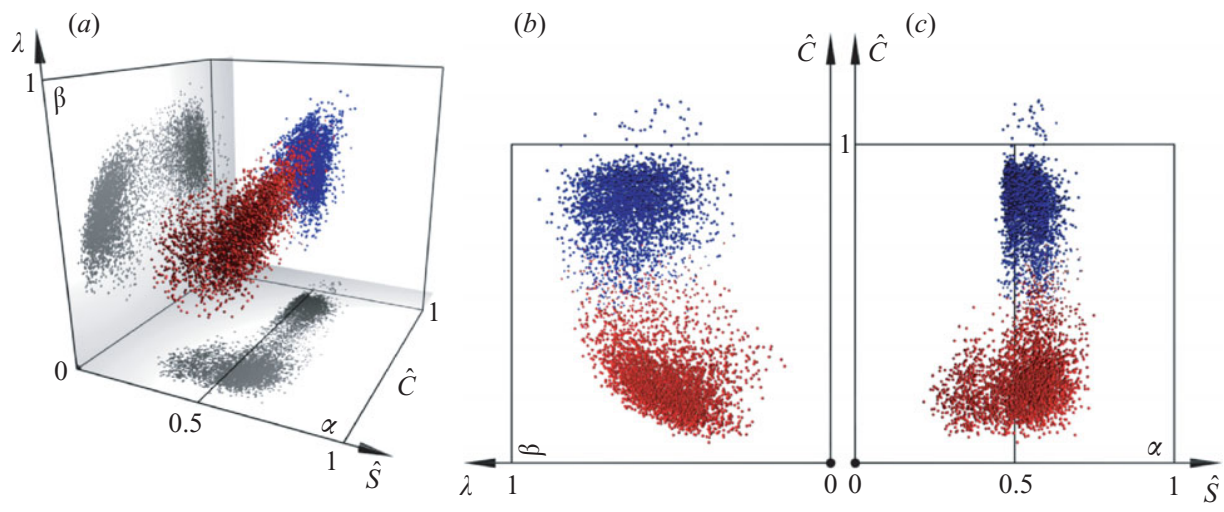

FIGURE 21. Clustering results in the visualization space - three-dimensional view $(a)$ and lateral $(b)$ and top $(c)$ projections - with glyphs (spheres) representing optimum clusters of structures educed from the set union of $Q$ and $\left[A_{i j}\right]_{+}$structures. Glyphs are scaled by the normalized silhouette value, which indicates the degree of membership of that element to the assigned cluster.

silhouette coefficient, a measure of the degree of membership of each structure to the cluster it was assigned. Comparing figures 21 and 20, it is confirmed that the two educed clusters correspond substantially to the two sets of structures $(Q$ and $\left.\left[A_{i j}\right]_{+}\right)$. Numerically, a matching of $96 \%$ between pairs of groups was obtained. A small percentage of structures of both fields shows a different geometry than the one expected according to the local criterion. For example, the central tube in figure 27 (discussed later) is a structure of $\left[A_{i j}\right]_{+}$, which is meant to educe sheet-like structures, while the other two tubes in that plot are structures of $Q$, as expected.

We emphasize that the scaling of the glyphs in figures 20 and 21 is different. Figure 20 uses the lognormalized area of each structure, and thus, it can be concluded, for both $Q$ and $\left[A_{i j}\right]_{+}$, that more stretched structures are typically larger (in area): glyphs with lower values of $\lambda$ (more stretched) are bigger in that figure. On the other hand, glyphs in figure 21 are scaled by the silhouette coefficient of the corresponding structure as a result of the clustering algorithm: bigger glyphs imply larger silhouette coefficient and, therefore, a higher degree of membership to the cluster the structure was automatically assigned. This different scaling contributes to an apparent lower density of glyphs in figure 20, when compared to figure 21, since glyphs associated with small structures would also be small in the former, while they will appear larger in the latter if they have a high silhouette coefficient.

Figure 22 shows the optimality scores found during the automatic determination of the number of clusters. It is observed that a number of clusters of 2 provides an optimality score near 0.7 (1.0 being the maximum), much higher, comparatively, than for other numbers of clusters. A comparison with the clustering results obtained for $\omega_{i} \omega_{i}$ and $S_{i j} S_{i j}$ in $\S 2$ (see figure 16), where the optimality scores were rather low and the variation among different number of clusters was small, gives an indication of the higher level of confidence in the clustering results for this case. This could be anticipated by looking at the organization of glyphs in the visualization space in figure 21, when compared to figure 15 .

Thus, the intuition that resulted by the visual cues of figure 19, in which isosurfaces of $Q$ seemed tube-like and structures of $\left[A_{i j}\right]_{+}$appeared sheet-like, has been 


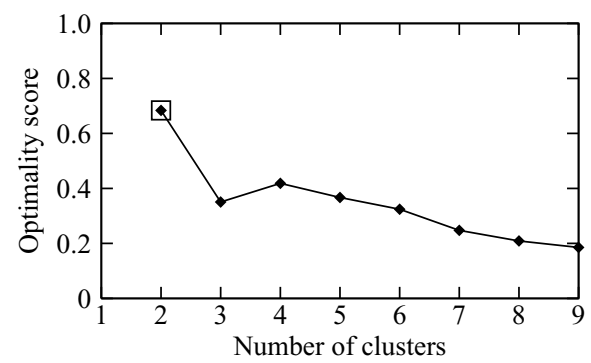

FIGURE 22. Optimality scores for different number of clusters obtained during the application of the clustering algorithm to the set of structures of $Q$ and $\left[A_{i j}\right]_{+}$together. An optimum number of clusters of 2 was automatically determined (squared point).

verified with a mathematical foundation first by the geometrical characterization, whose results can be partially seen in figure 20, and then by the application of clustering techniques to the union of both sets, whose results are seen in figure 21 . The geometrical data can be used both qualitatively and quantitatively.

\section{Interaction among structures of different fields: proximity issues}

This section is devoted to the study of the interplay among structures of different fields under a geometrical perspective. We present here a methodology that, for each individual structure, performs an analysis of its proximal structures based upon their geometrical characterization previously introduced. Statistical results are obtained for the set of structures under study, presented in the form of combined probability density functions.

A motivation for this methodology is presented in $\S 4.1$, followed by the description of the methodology itself in $\S 4.2$. Results of its application to the four fields studied in previous sections of this paper grouped by pairs, namely $Q-\left[A_{i j}\right]_{+}$and $\omega_{i} \omega_{i}-S_{i j} S_{i j}$, are presented in $\S \S 4.3$ and 4.4 , respectively.

\subsection{Motivation}

The scalar fields that have been studied in the previous sections were all derived from the velocity-gradient tensor. Some of them can be formulated in terms of the others by simple algebraic relations (e.g. $Q$ in terms of $\omega_{i} \omega_{i}$ and $S_{i j} S_{i j}$ ). Other fields, not studied here but common in the study of turbulence, such as the pressure field, $p$, are also related to these (see (3.3)). A passive scalar field could also be added to the flow and related to the other fields through the advection-diffusion equation. They all can be thought of as different manifestations of the same flow, in this case, incompressible homogeneous isotropic turbulence decaying in time in a periodic box. The mathematical relations among all of them are well known, and a vast effort has been dedicated in the fluid mechanics literature to study how those mathematical relations are translated into the physical aspects of turbulence, both in physical and Fourier domains, through the study of pdfs, structure and (auto-)correlation functions, spectra, etc.

It is thus conceivable that the structures extracted from those fields may have some relations; first, in their relative locations in physical space and, second, in their geometrical character, forming composite structures localized in a physical domain. Perhaps the most common example of such inter-relation between scalar fields is the formation process of a vortex tube that results from the roll-up of a vortex sheet (see 
(a)

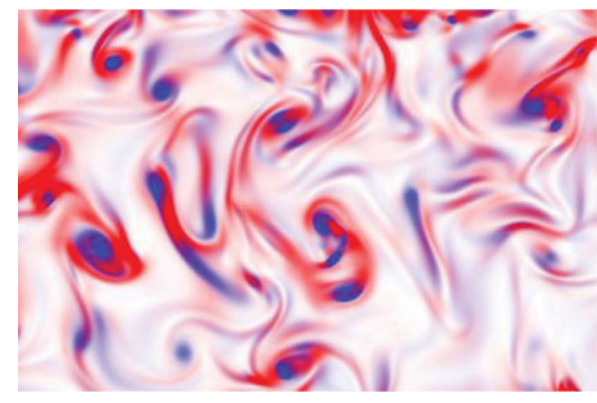

(b)

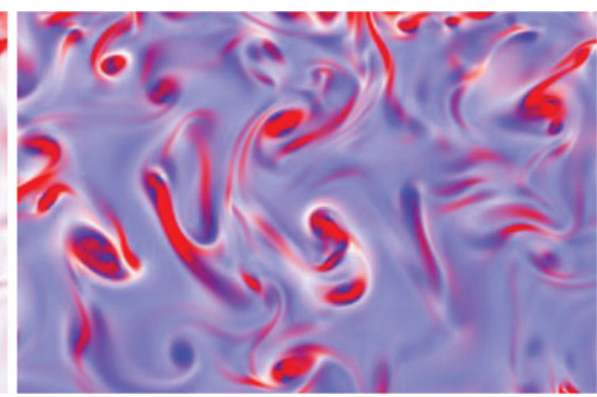

Figure 23. ( $a$ ) Plane cut of $S_{i j} S_{i j}$ (red) superimposed over equivalent plane cut of $\omega_{i} \omega_{i}$ (blue). (b) Plane cut of $\left[A_{i j}\right]_{+}$(red) superimposed over equivalent plane cut of $Q$ (blue).

$\mathrm{HF}$ and the references therein): at an intermediate stage of that process, the core of the vortex tube is dominated by high values of vorticity, while the sheet that is rolling up around it presents high values of dissipation. Therefore, in that scenario, tube-like structures of $\omega_{i} \omega_{i}$ would be surrounded by sheet-like structures of $S_{i j} S_{i j}$. Similarly, considering the scalar fields used by the local identification criteria in $\S 3.1$ to educe vortex tubes and sheets, structures of $Q$ (which were found to be tube-like) would be surrounded by structures of $\left[A_{i j}\right]_{+}$(predominantly sheet-like).

Plane cuts of pairs of scalar fields, taken at the same location, are superimposed in figure $23\left(S_{i j} S_{i j}\right.$ over $\omega_{i} \omega_{i}$ on the left, $\left[A_{i j}\right]_{+}$over $Q$ on the right). Close relations between their corresponding structures are noticed: structures of $S_{i j} S_{i j}$ and $\left[A_{i j}\right]_{+}$ (red) tend to wrap around those of $\omega_{i} \omega_{i}$ and $Q$ (blue), respectively. It is also observed that many structures of $\omega_{i} \omega_{i}$ and $Q$ appear to have circular cores, while structures of $S_{i j} S_{i j}$ and $\left[A_{i j}\right]_{+}$are more elongated. When extrapolated to the three-dimensional fields, those circular patches of the plane cuts of $\omega_{i} \omega_{i}$ and $Q$ will likely belong to tubes, while the elongated regions of plane cuts of $S_{i j} S_{i j}$ and $\left[A_{i j}\right]_{+}$will probably correspond to sheets around them. Another scenario in which circular and elongated regions of the plane cuts correspond, respectively, to blob-like and tube-like structures in three dimensions would be also possible, but its frequency of occurrence is comparatively smaller, as concluded from the study of the geometry of the four fields done in $\S 2$. A methodology-enabling study, in three dimensions, of the geometry of structures of different fields surrounding those of a particular field would be useful to test this visual intuition and to quantify its appearance.

\subsection{Methodology}

Consider two sets of structures, $\mathscr{A}$ and $\mathscr{B}$, containing $N_{\mathscr{A}}$ and $N_{\mathscr{B}}$ elements, respectively. We impose no Boolean restriction on both sets, so that elements of $\mathscr{A}$ can also be elements of $\mathscr{B}$.

\subsubsection{Processing individual structures}

For each structure $a_{i} \in \mathscr{A}$ :

(a) Obtain the subset $\mathscr{C}$ of $N_{\mathscr{C}}$ structures of $\mathscr{B}(\mathscr{C} \subseteq \mathscr{B})$ that are closest to $a_{i}$ in the bounding-box sense. This step is intended to speed up the algorithm by reducing the load required to perform the rest of the steps.

(b) For each $c_{j} \in \mathscr{C}, j=1, \ldots, N_{\mathscr{C}}$, obtain the point-wise minimum distance map from $c_{j}$ to $a_{i}, M D M\left(c_{j}, a_{i}\right)$. This map computes, for each point of the discretized surface $c_{j}$, the minimum of the distances from it to all points of $a_{i}$. 


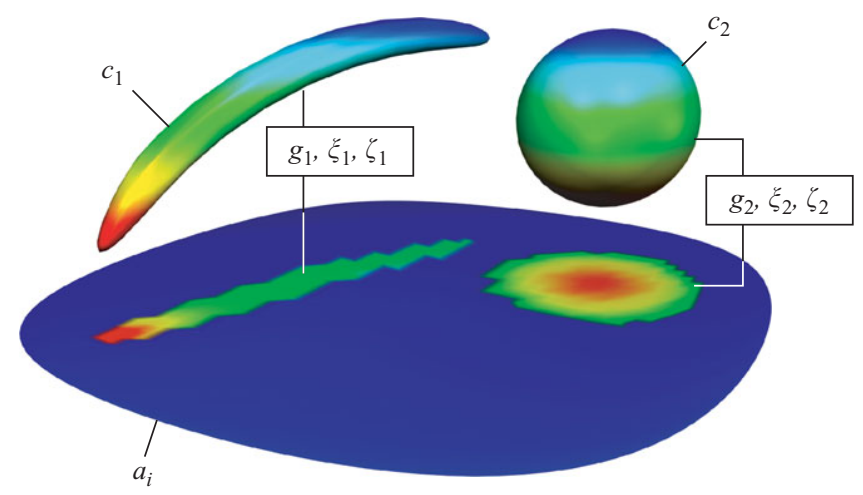

FIGURE 24. Schematic of the computation of the conditional array map (CAM) for a given structure $a_{i}$ (sheet-like) with respect to two close structures $c_{1}$ (tube-like) and $c_{2}$ (blob-like). Minimum distance maps from $c_{j}(j=1,2)$ to $a_{i}$ are shown, with a common colour scale for both ranging from red to blue, representing smaller to larger minimum distances to $a_{i}$, respectively. The proximity value derived from them is mapped on $a_{i}$, with a colour scale varying from blue (nil proximity) to red (maximum proximity). Those points of $a_{i}$ tagged during the computation of the minimum distance maps (with proximity values greater than zero) will also store the values $\left\{g_{j}, \xi_{j}, \zeta_{j}\right\}$ of the corresponding $c_{j}$ in the conditional array map.

At the same time, during the computation of $M D M\left(c_{j}, a_{i}\right)$, those points of $a_{i}$ that provide the minima for $M D M\left(c_{j}, a_{i}\right)$ are tagged and an array of $N_{\gamma}$ parameters is stored, for each one of those points, in a point-wise conditional array map of $a_{i}, C A M\left(a_{i}\right)$. The array of parameters $\left\{\gamma_{k}, k=1, \ldots, N_{\gamma}\right\}$ contains, for each tagged point $P \in a_{i}$, the (dimensionless) proximity value, $p$, obtained from the minimum distance, $d$, to the point(s) of $c_{j}$ for which $P$ was the closest of all points in $a_{i}$, plus additional information of $c_{j}$ itself (for example, but not limited to, an identifier of the structure $c_{j}$, and geometrical parameters of $c_{j}$ obtained from a previous characterization performed on it). The dimensionless proximity, $p$, is defined in terms of the distance $d$ by $p \equiv(1+d / D)^{-1}$, where $D$ is a non-dimensionalizing length-scale of $a_{i}$. Smaller distances, $d$, translate into higher proximity values $(p \in[0,1])$.

We consider here the particular case in which, for each point $P$ of $a_{i}$, the array $\left\{\gamma_{k}, k=1, \ldots, N_{\gamma}\right\}$ consists of four parameters $\left(N_{\gamma}=4\right)$, redefined for simplicity as $\{p, g, \xi, \zeta\}_{P}: p$ is the dimensionless proximity value to $c_{j}$ described above, $g$ is an index categorizing $c_{j}$ among $N_{g}$ known groups of structures present in $\mathscr{B},\left\{G_{g} \subset\right.$ $\left.\mathscr{B}, g=1, \ldots, N_{g} \| \bigcup_{g=1}^{N_{g}} G_{g}=\mathscr{B}\right\}, \xi$ and $\zeta$ are geometrical properties of $c_{j}$. A schematic of the computation of the conditional array map for $a_{i}$ based on the minimum distance maps for $c_{j}$ is depicted in figure 24 .

As different $c_{j}$ are processed, the conditional array map for $a_{i}, C A M\left(a_{i}\right)$, is updated at previously untagged points of $a_{i}$. Also, the array of parameters of a previously tagged point of $a_{i}$ is updated if the new proximity value for that point is larger than the previously stored one, meaning that the corresponding distance of the new structure $c_{j}$ to that point is smaller than all the previous $c_{j}$ structures processed.

If $c_{j}$ is the same structure as $a_{i}$, which can happen, since, as we stated above, $\mathscr{A}$ and $\mathscr{B}$ could share elements, then it is discarded in the computation of $\operatorname{CAM}\left(a_{i}\right)$. Otherwise it would eclipse all other structures of $\mathscr{C}$ and the conditional array map would be trivial and useless. Nevertheless, if $c_{j}$ is not the same as $a_{i}$, but both happen to be identical, then $c_{j}$ is included in the computation of $C A M\left(a_{i}\right)$, resulting in a 
trivial but useful result. For example, if $\mathscr{A}$ and $\mathscr{B}$ are disjoint but their structures are identical by pairs $\left\{a_{i}, b_{i}\right\}$, the trivial $C A M\left(a_{i}\right)$ obtained for each $a_{i}$ after processing all structures $c_{j} \in \mathscr{C}$ would prove their identicality.

(c) After all $c_{j}$ have been processed for a given $a_{i}$, we obtain from the $\operatorname{CAM}\left(a_{i}\right)$ the joint probability density function (jpdf), in terms of $p$ and $g$, based on area coverage, denoted by $\left.\mathscr{P}(p, g)\right|_{a_{i} \leftarrow \mathscr{C}}$. The value $\left.\int_{p_{1}}^{p_{2}} \mathscr{P}(p, g)\right|_{a_{i} \leftarrow \mathscr{C}} \mathrm{d} p$ can be interpreted as the probability of finding structures of $\mathscr{C}$ categorized in a group $G_{g}$ as being the closest structures to $a_{i}$ in the range of proximity values $\left[p_{1}, p_{2}\right]$. At a given point $P \in a_{i}$, a structure $c_{j^{*}}$ is the closest to $a_{i}$ at $P$ if it has the highest value of proximity, $p$, among all the structures $c_{j}$ that would tag $P$ in the computation of $C A M\left(a_{i}\right)$.

We also compute the area-based joint probability density function in terms of $\xi$ and $\zeta$, with an additional intensity component based on the averaged proximity value. For each $2 D$ interval of the geometrical properties $\left[\xi_{m}, \xi_{m}+\Delta \xi\right] \times\left[\zeta_{n}, \zeta_{n}+\Delta \zeta\right]$, the discrete joint probability density function with intensity $(\mathrm{jpdf}+\mathrm{i})$ has two components: the first one is the pdf value itself, i.e. surface area of the $a_{i}$ such that $(\xi, \zeta) \in$ $\left[\xi_{m}, \xi_{m}+\Delta \xi\right] \times\left[\zeta_{n}, \zeta_{n}+\Delta \zeta\right]$ divided by the total area of $a_{i}$; the second component is the area-weighted average of the proximity values of all faces of the discretized $a_{i}$ such


where the vector symbol reflects that it has two components (pdf in terms of $\{\xi, \zeta\}$ and intensity based on $p$ ). We approximate:

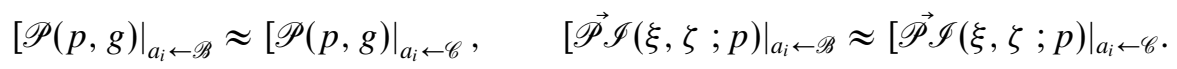

This approximation is exact when the structures of $\mathscr{C}$ eclipse, for $a_{i} \in \mathscr{A}$, structures of $\mathscr{B}-\mathscr{C}$, if $N_{\mathscr{C}}<N_{\mathscr{B}}$, and also in the trivial case $N_{\mathscr{C}}=N_{\mathscr{B}}$, for which it becomes an identity.

\subsubsection{Transition from individual structures to results for the set $\mathscr{A}$}

Once all $a_{i} \in \mathscr{A}$ have been processed, global jpdf and jpdf $+\mathrm{i}$ are obtained for the set $\mathscr{A}$ as the average of individual jpdf and jpdf+i for each $a_{i}$ :

$$
\begin{aligned}
& {\left[\left.\mathscr{P}(p, g)\right|_{\mathscr{A} \leftarrow \mathscr{B}}=\frac{\sum_{a_{i} \in \mathscr{A}}\left[\left.\mathscr{P}(p, g)\right|_{a_{i} \leftarrow \mathscr{B}}\right.}{N_{\mathscr{A}}},\right.}
\end{aligned}
$$

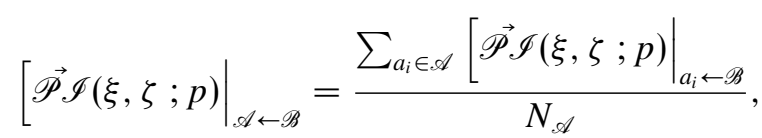

respectively. This is equivalent to assigning a probability density of $\left[\left.\mathscr{P}(p, g)\right|_{a_{i} \leftarrow \mathscr{B}} / N_{\mathscr{A}}\right.$ to each event $a_{i} \leftarrow \mathscr{B}$ and then computing the probability density of the event $\mathscr{A} \leftarrow \mathscr{B}$ as the union of all individual events $\left(\forall a_{i} \in \mathscr{A}\right)$, taking them as independent. The same reasoning applies to $\left[\left.\overrightarrow{\mathscr{P} \mathscr{I}}(\xi, \zeta ; p)\right|_{\mathscr{A} \leftarrow \mathscr{B}}\right.$.

\subsubsection{Computational remarks}

When dealing with the discretized surfaces representing the structure $a_{i}$, the jpdfs $\left[\left.\mathscr{P}(p, g)\right|_{a_{i} \leftarrow \mathscr{B}} / N_{\mathscr{A}}\right.$ and $\left[\left.\vec{P} \mathscr{I}(\xi, \zeta ; p)\right|_{\mathscr{A} \leftarrow \mathscr{B}}\right.$ are obtained from face-wise data, and not from point-wise data. This requires a transformation from point-wise to face-wise data, which is done in this case by assigning to each face the array $\left\{\gamma_{k}, k=1, \ldots, N_{\gamma}\right\}$ of its vertex with the maximum value of the proximity (i.e. minimum distance). Interpolation, in this case, is inappropriate since there is no guarantee of continuity of the values of the parameters $\gamma_{k}$ throughout the surface, as neighbouring points can 
have data corresponding to different surrounding structures $c_{j} \in \mathscr{C}$. When continuity of the jpdf variables exists, an interpolation (for example, tri-linear if the faces are triangular) is justified and can improve the accuracy of the jpdf, relaxing the dependence between the discretization of the surface and the discretization of the jpdf; this was the case in the computation of the $\mathscr{P}(S, C)$ used to obtain the signatures of the structures in the characterization step of the study of the non-local geometry of structures, but cannot be applied to the jpdfs we are analyzing in this section.

The algorithm can be modified to obtain directly face-wise data instead of pointwise data and avoid such transformation. Point-wise data has been chosen in our implementation due to the simplicity and increased speed when computing distance maps. Nonetheless, we note that this imposes the additional constraint that both discretized surfaces $a_{i}$ and $c_{j}$ must have an equivalent grid resolution for an accurate computation of the distance maps, basis of this algorithm. In our implementation, this is guaranteed as a consequence of the iso-contouring algorithm in use and the fact that, even when multi-scale techniques were applied, sub-sampling was not performed on the grid for any scale, and no decimating operation was applied over the discretized structures thereafter.

An example of application of the methodology described above is introduced in Appendix C. It is developed analytically and then a numerical application is shown.

\subsection{Application to structures of $Q$ and $\left[A_{i j}\right]_{+}$}

We apply the methodology explained in $\S 4.2$ to the structures of the fields $Q$ and $\left[A_{i j}\right]_{+}$educed in $\S 3.1$, for the $1024^{3}$ grid resolution. In our first application, the set $\mathscr{A}$ will be composed of the extracted structures of $Q, \mathscr{A} \equiv \mathscr{X}(Q)$, where $\mathscr{X}(\alpha)$ denotes the set of extracted structures from a three-dimensional scalar field $\alpha$, while the set $\mathscr{B}$ is composed of the union of structures extracted from $Q$ and those extracted from $\left[A_{i j}\right]_{+}, \mathscr{B} \equiv \mathscr{X}(Q) \cup \mathscr{X}\left(\left[A_{i j}\right]_{+}\right)$. Therefore, in this particular case, $\mathscr{A} \subset \mathscr{B}$.

We choose $(\xi, \zeta)$ as the geometrical parameters $(\hat{S}, \hat{C})$ obtained in the characterization step of the non-local study of the geometry of structures applied in $\S 3.2$. The index $g$ refers in this case to the two groups of structures in which $\mathscr{B}$ can be immediately divided, namely, structures of $Q(g=1)$ and structures of $\left[A_{i j}\right]_{+}$ $(g=2)$. The length-scale $D$ used to non-dimensionalize distances when computing proximity values for each structure $a_{i}$ is taken to be the parameter $\mu \equiv 3 \mathrm{~V} / \mathrm{A}$, where $V$ is the volume and $A$ is the area of $a_{i}$. Note that this parameter was used in the geometrical characterization step of BP to non-dimensionalize the curvedness (see (2.2)), resulting in the dimensionless value $C$, from which $\hat{C}$ was obtained. For a sphere of radius $R, \mu_{\text {sphere }}=R$; for an elongated tube with circular cross-section of radius $R, \mu_{\text {tube }} \approx 3 R / 2$; for a predominantly sheet-like structure of thickness $t$, $\mu_{\text {sheet }} \approx 3 t / 2$. After applying the methodology, we obtain both $\left[\left.\vec{P} \mathscr{I}(\hat{S}, \hat{C} ; p)\right|_{\mathscr{A} \leftarrow \mathscr{B}}\right.$ and $\left[\left.\mathscr{P}(p, g)\right|_{\mathscr{A} \leftarrow \mathscr{B}}\right.$.

\subsubsection{Proximity and area coverage of surrounding structures through jpdf $+i$}

Figure 25 shows $\left[\left.\overrightarrow{\mathscr{P} \mathscr{I}}(\hat{S}, \hat{C} ; p)\right|_{\mathscr{A} \leftarrow \mathscr{B}}\right.$. Figure $25(a)$ plots the representation of the pdf component, using a greyscale, where white indicates nil area coverage and black indicates the maximum area coverage. Therefore, dark regions indicate that structures of $\mathscr{B}$ with values of $(\hat{S}, \hat{C})$ within those regions are found, on average, to surround comparatively larger proportion of the area of structures of $\mathscr{A}$. Light regions indicate values of $(\hat{S}, \hat{C})$ not so commonly encountered in the structures of $\mathscr{B}$ surrounding those of $\mathscr{A}$. Figure $25(b)$ represents the intensity component, which in this case corresponds to the proximity value, using a colour-scale (continuous gradient 
(a)

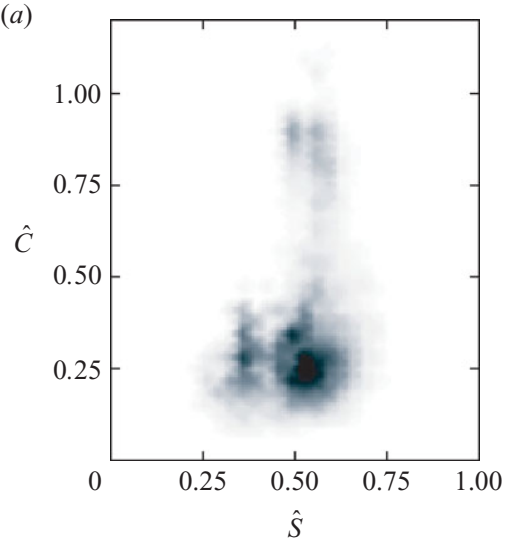

area coverage pdf

(c)

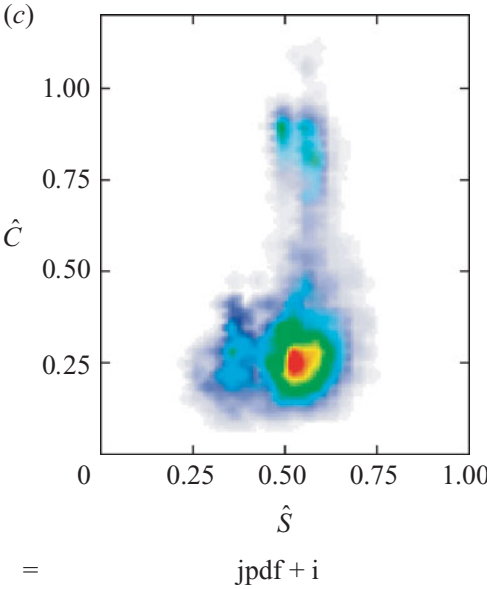

(b)

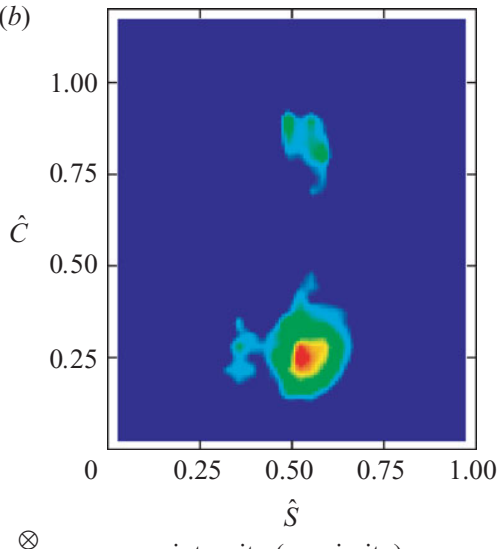

(d)

with

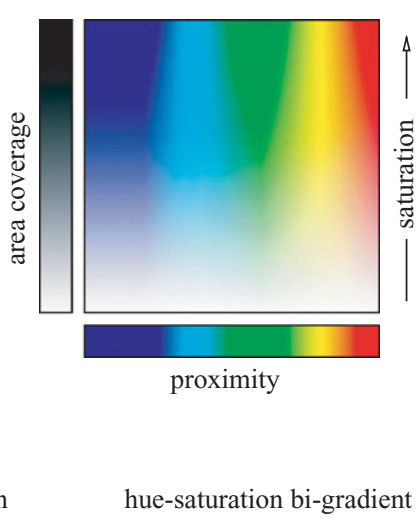

intensity (proximity)

FIGURE 25. Components of the jpdf $+\mathrm{i}$ in terms of $(\hat{S}, \hat{C})$ plus intensity component based on proximity, of structures of $\mathscr{X}(Q) \cup \mathscr{X}\left(\left[A_{i j}\right]_{+}\right)$surrounding structures of $\mathscr{X}(Q)$ : area coverage pdf component $(a)$ using greyscale; intensity component $(b)$ using blue-cyan-green-yellow-red colour-scale; composition of area coverage pdf and intensity components to obtain the composite plot $(c)$ with bi-dimensional hue-saturation gradient scale $(d)$ corresponding to proximity (hue) and area coverage (saturation).

blue-cyan-green-yellow-red): blue indicates low proximity (farther distances) while red indicates high proximity (closer distances) of the structures of $\mathscr{B}$ with given values $(\hat{S}, \hat{C})$ to those of $\mathscr{A}$. Figure $25(c)$ plots a composition of both the pdf component and the intensity component by means of a hue-saturation gradient, represented in figure $25(d)$. In this HSB (hue, saturation, brilliance) colour space, the area coverage corresponds to the saturation, $S$, while the intensity component corresponds to the hue, $H$, and the brilliance, $B$, is kept constant at its maximum value. Therefore, it contains information of the averaged area coverage in the saturation scale and information of the averaged proximity in the hue scale. Saturated-red regions correspond to values of $(\hat{S}, \hat{C})$ found in structures of $\mathscr{B}$ closest to those of $\mathscr{A}$ and covering, comparatively, the largest proportion of their surface area. Desaturated regions indicate less area coverage and colours closer to the blue hue indicate lower values of the proximity (and thus, farther structures). Regions of saturated-blue, for example, will indicate 


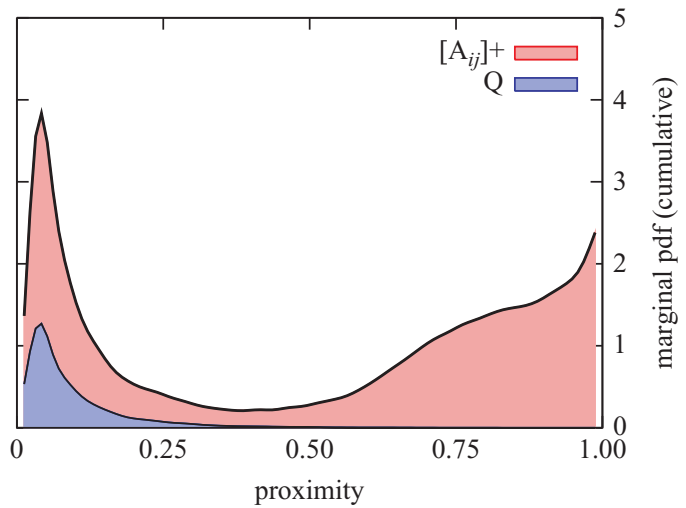

Figure 26. Representation of the marginal pdf of $\left[\left.\mathscr{P}(p, g)\right|_{\mathscr{A} \leftarrow B}\right.$ in terms of the proximity $p$ (thick black line), showing the contribution of each group $g$ by the different coloured areas between two black lines (added cumulatively).

that structures of $\mathscr{B}$ with those values of $(\hat{S}, \hat{C})$ appear far but cover a large proportion of the surface area of structures of $\mathscr{A}$. Desaturated blue regions, on the other hand, will indicate that structures of $\mathscr{B}$ with those values of $(\hat{S}, \hat{C})$ appear far as well, but covering a small fraction of the surface area of structures of $\mathscr{A}$. Note that both scales are normalized: the maximum area coverage will have a saturated colour, and red hue corresponds to the maximum proximity value. Therefore, they provide only relative (not absolute) information of the area coverage and the intensity (proximity) values. This can be changed fixing absolute ranges for the hue-saturation bi-gradient scale.

The first conclusion that can be directly drawn from figure 25 is that structures of $Q$ are mainly surrounded (more saturation), among those of $\left[A_{i j}\right]_{+}$and $Q$ itself, by structures with low values of $\hat{C}$, which are also closer (red hues). Those $\hat{C}$ values correspond to sheet-like structures. A desaturated region (less area coverage) of green/cyan hues (farther structures) is located near the $(\hat{S}, \hat{C}) \approx(1 / 2,1)$ zone. This implies that, secondarily, tube-like structures also surround structures of $Q$, but they are not so proximal and cover a smaller relative surface area of them. When figure 25 is compared to figure $20(c)$, since both share the same axes $(\hat{S}, \hat{C})$, it is indirectly concluded that the majority of sheet-like structures surrounding those of $Q$ (as seen in figure 25 by the saturated red-coloured regions) are structures of $\left[A_{i j}\right]_{+}$since they are the ones with a higher density of glyphs in the corresponding regions in the $\hat{S}-\hat{C}$ plane of figure 20 .

\subsubsection{Proximity split by groups through cumulative marginal $p d f s$}

In figure 26, we plot cumulative one-dimensional marginal pdfs obtained from $\left[\left.\mathscr{P}(p, g)\right|_{\mathscr{A} \leftarrow B}\right.$ for increasing group $g$ numbers, $\left\{f_{k}(p)=\sum_{g=1}^{k}\left[\left.\mathscr{P}(p, g)\right|_{\mathscr{A} \leftarrow B}, k=\right.\right.$ $\left.1, \ldots, N_{g}\right\}$. The contribution of each group $g$ is represented by a different colour between black lines. For $k=N_{g}$, the result is the marginal pdf in terms of $p$, $f(p)=f_{N_{g}}(p)$, represented by a thicker black line. From figure 26 , it is directly concluded that structures of $Q$ are predominantly surrounded by structures of $\left[A_{i j}\right]_{+}$, represented by the red area of the marginal pdf. The blue area corresponds to structures of $Q$, which cover a much smaller fraction and are farther (lower values of proximity) than those of the majority of $\left[A_{i j}\right]_{+}$structures. This was indirectly concluded previously from the combination of figures 25 and 20. The contribution of 


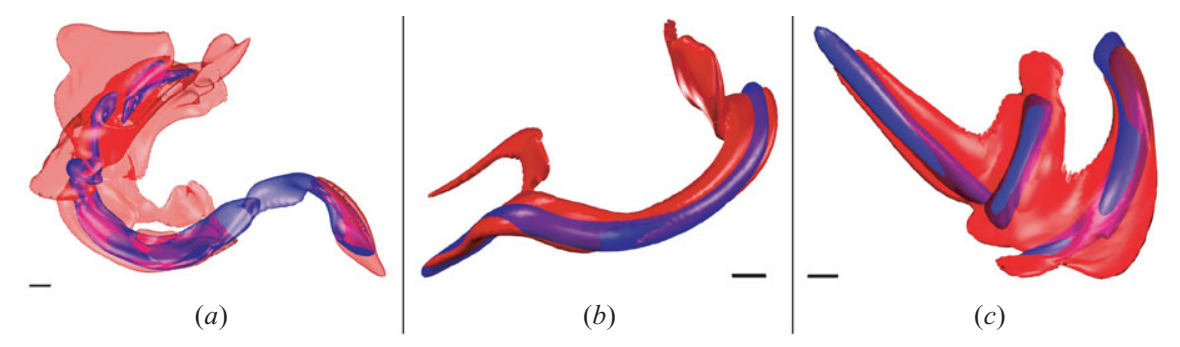

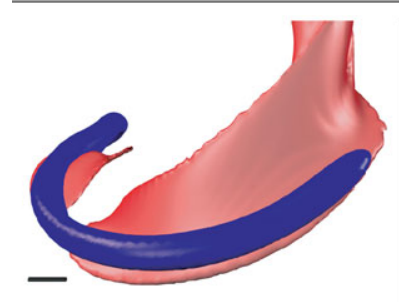

(d)

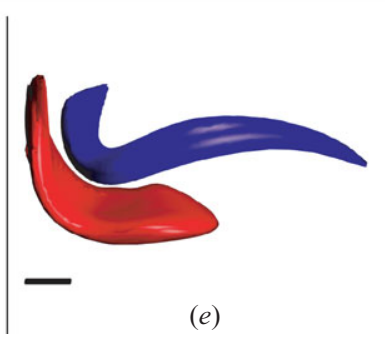

(e)

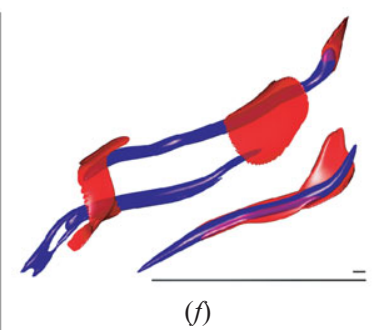

$(f)$

FIGURE 27. Examples of composite structures formed by interaction of tube-like (blue) and sheet-like (red) geometries found in the database. In cases $(a)$ and $(b)$, a tube is mostly embedded by one or more sheets that intersect it and remain at a close distance. Case (c) consists of three tubes and a sheet that follows closely their geometry, intersecting them, and connects them through stretched regions. Note how two of the three tubes are nearly transversely aligned: similar configurations of tubes and sheets were often found in HF, where they proposed a creation process based on the interaction of the sheets surrounding the tubes. Cases $(d)$ and $(e)$ show, each, a sheet-like structure wrapping around a tube, without intersecting it but remaining at a close distance and following its curvature. A smaller sheet intersecting the tube is also seen in case $(d)$. Case $(f)$ shows three tubes at moderate distances from each other $(\approx 5-10$ times their average radius $)$ with a similar orientation and sheets partially surrounding them at a close distance or even intersecting them. For clarity, only a subset of all the nearby structures surrounding each tube in every case is shown. The length of the small horizontal black bars at the bottom of each plot represents the average Kolmogorov scale, $\bar{\eta}$, for reference. In addition, for case $(f)$, the Taylor microscale is also represented by the length of the longer black bar at the bottom.

$\left[A_{i j}\right]_{+}$(red area) to the marginal pdf in figure 26 shows four regions of interest; first, the region near unitary proximity $(p \approx 1)$, which corresponds to structures of $\left[A_{i j}\right]_{+}$ very close to those of $Q$, likely overlapping/intersecting each other. Examples of this interaction, extracted from the database under study, are shown in figure 27, cases $(a),(b)$ and $(c)$. Second, the region with proximity values between 0.6 and 0.9 , where a hump is visible in the marginal pdf, which corresponds to structures of $\left[A_{i j}\right]_{+}$(or parts of them) still at a close distance but not overlapping or intersecting. Those values of proximity translate into distances between $1 / 10$ and $2 / 3$ times their own characteristic length, defined by $\mu$, which corresponds, for the tube-like structures predominant in $Q$, to approximately $3 / 2$ times their characteristic radius. See cases $(d),(e)$ and sheets surrounding tubes in case $(f)$ of figure 27 for some examples of this configuration. Third, the region with proximity values between 0.25 and 0.5 with low values of the marginal pdf indicates that surrounding structures are rarely found at those distances. The fourth region corresponds to low values of proximity $(p \approx 0.1-0.2)$. See, for example, tubes nearby other tubes in case $(f)$ of figure 27 . We note, as a result of the definition of $p$, that a wide range of high values $(>4)$ of the relative distance between structures accumulate in that region of low $p$. Thus, it is expected to find 

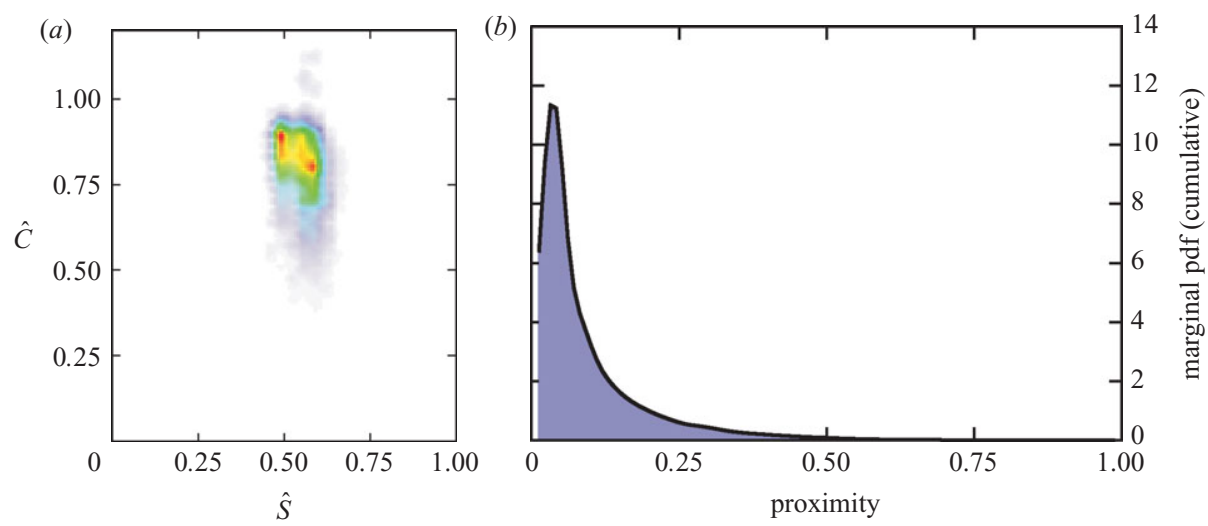

FIGURE 28. Results for structures of $Q$ surrounding themselves: $\left[\left.\vec{P} \mathscr{I}(\hat{S}, \hat{C} ; p)\right|_{\mathscr{A} \leftarrow \mathscr{B}}\right.$ (a) and $\left[\left.\mathscr{P}(p, g)\right|_{\mathscr{A} \leftarrow \mathscr{B}}(b)\right.$ for the case $\mathscr{A}=\mathscr{B}=\mathscr{X}(Q), g=1$.

an accumulation of the marginal pdf for low $p$ whenever structures of $\mathscr{A}$ are not completely surrounded by close ones of $\mathscr{B}$. A modification of the definition of $p$ or the scale used to represent it can spread the effect of those higher distance over a wider range if required, but here we are more interested in closer structures, so the current $p$ seems suitable for this purpose.

\subsubsection{Structures of $Q$ surrounding themselves}

A question that arises after this analysis is whether structures of $Q$ might be closer to themselves than what the blue region in figure 26 shows, but structures of $\left[A_{i j}\right]_{+}$eclipsing them when computing $\left[\left.\overrightarrow{\mathscr{P}} \mathscr{I}(\hat{S}, \hat{C} ; p)\right|_{\mathscr{A} \leftarrow \mathscr{B}}\right.$ masquerade the result, making them appear farther than they are. This can be answered by applying the proposed methodology to $\mathscr{A}=\mathscr{B}=\mathscr{X}(Q)$, that is, considering the problem of how structures of $Q$ surround themselves. Figure 28 shows the $\left[\left.\overrightarrow{\mathscr{P} \mathscr{I}}(\hat{S}, \hat{C} ; p)\right|_{\mathscr{L} \leftarrow \mathscr{B}}(a)\right.$ and $\left[\left.\mathscr{P}(p, g)\right|_{\mathscr{A} \leftarrow \mathscr{B}}(b)\right.$ obtained in this case. Note that now there is only one group $(g=1)$. $\left[\left.\overrightarrow{\mathscr{P}} \mathscr{I}(\hat{S}, \hat{C} ; p)\right|_{\mathscr{A} \leftarrow \mathscr{B}}\right.$ shows, as expected, tube-like geometries as the proximal, which is trivial once the sheet-like structures of $\left[A_{i j}\right]_{+}$have been removed from $\mathscr{B}$. But, $\left[\left.\mathscr{P}(p, g)\right|_{\mathscr{A} \leftarrow \mathscr{B}}\right.$ also shows a peak at about the same value of proximity that was found for $Q$ structures when $\left[A_{i j}\right]_{+}$structures were included, which confirms the farther distances among $Q$ structures to themselves. See case $(f)$ in figure 27.

\subsection{Application to structures of $\omega_{i} \omega_{i}$ and $S_{i j} S_{i j}$}

Now we apply this methodology to structures of $\omega_{i} \omega_{i}$ and $S_{i j} S_{i j}$ educed in $\S 2.3$. For these two fields, a multi-scale decomposition was performed. Of all possible combinations of fields and scales, we study two cases due to their particular relevance. In both cases, we take $\mathscr{A}$ as the set of structures of $\omega_{i} \omega_{i}$ at scale number 3 (intermediate scale). They were found in $\S 2.4$ to be predominantly tube-like. We take $\mathscr{B}$ as the set of structures educed for scales numbers 3-6 for $\omega_{i} \omega_{i}$ in the first case (thus, $\mathscr{A} \subset \mathscr{B}$ ) and for $S_{i j} S_{i j}$ in the second (thus, $\mathscr{A} \cap \mathscr{B}=\emptyset$ ). In both cases, we split $\mathscr{B}$ into four groups $\left(N_{g}=4\right)$, each corresponding to a different scale number of the field under consideration $\left(\omega_{i} \omega_{i}\right.$ or $S_{i j} S_{i j}$, respectively).

Figure 29 shows $\left[\left.\overrightarrow{\mathscr{P}} \mathscr{I}(\hat{S}, \hat{C} ; p)\right|_{\mathscr{A} \leftarrow \mathscr{B}}\right.$ (left) and $\left[\left.\mathscr{P}(p, g)\right|_{\mathscr{A} \leftarrow \mathscr{B}}\right.$ (right), for the first $(a)$ and second $(b)$ cases. From the jpdf $+\mathrm{i}$ (left plots), it is observed that structures with small $\hat{C}$ (corresponding to sheet-like geometries) appear to be the closest in both 



FIGURE 29. Results for structures of $\omega_{i} \omega_{i}$ at scale number 3 surrounded by structures of $\omega_{i} \omega_{i}(a)$ and by structures of $S_{i j} S_{i j}(b)$, at scale numbers $3-6:\left[\left.\overrightarrow{\mathscr{P} \mathscr{I}}(\hat{S}, \hat{C} ; p)\right|_{\mathscr{A} \leftarrow \mathscr{B}}\right.$ (left) and $\left[\left.\mathscr{P}(p, g)\right|_{\mathscr{A} \leftarrow \mathscr{B}}\right.$ (right).

cases (yellow and red spots). But, there is a wide range of geometries among the surrounding structures.

In the first case, that is, for $\mathscr{B}$ containing structures of $\omega_{i} \omega_{i}$, two saturated regions (implying high area coverage) are present in that plot, corresponding to tube-like (nearer the $(\hat{S}, \hat{C}) \approx(1 / 2,1)$ region) and sheet-like (small $\hat{C}$ values) structures. The latter appear closer (red and yellow hues, as opposed to green and cyan), but the former seem to cover a slightly higher percentage of the area of structures of $\mathscr{A}$ (more saturated colours). The region in between those two (intermediate values of $\hat{C}$ ) contains structures which appear farther and cover a smaller area.

In the second case, $\mathscr{B}$ containing $S_{i j} S_{i j}$ structures, the highest area coverage corresponds clearly to those structures with low $\hat{C}$ (sheet-like), which are also the closest (red, yellow and green hues). The spread towards other geometries is also significant, but the area coverage and proximity decreases in those other regions of the $\hat{S}-\hat{C}$ plane (less saturation and cyan and blue hues). Also, the region around tube-like structures found in the first case is now, in the second case, more diffuse and spread towards the blob-like region. This is consistent with the fact that less tubes were found in $\S 2.3$ in the geometrical analysis of structures of $S_{i j} S_{i j}$.

The right plots of figure 29 show the cumulative marginal pdf obtained from $\left[\left.\mathscr{P}(p, g)\right|_{\mathscr{A} \leftarrow \mathscr{B}}\right.$, split by groups corresponding to each scale number under study (3-6). 
We use the same colour code for each scale number as in figures 8-13. There are two main differences between both cases of study. In the first case, structures of $\omega_{i} \omega_{i}$ at scale number 3 have a small contribution to the marginal pdf, and appear far $(p \approx 0.1)$ compared to the rest of the scales; structures at scale number 4 are the closest, showing a significant increase of $p$ in the range $0.6-0.8$, while structures at scale numbers 5 and 6 also appear close, but with a more gradual increase for $p>0.6$. In the second case, structures of $S_{i j} S_{i j}$ at each one of the scales under analysis (3-6) have a more balanced contribution to the marginal pdf; scale number 3 shows a slightly higher concentration of closer structures $(p>0.6)$, and less proportion of farther structures $(p \approx 0.1)$ when compared to structures at scale numbers 4-6.

\subsection{Discussion}

From this proximity study, tubes appear closely surrounded by sheets, when both structures of $Q$ and those of an intermediate scale of $\omega_{i} \omega_{i}$ are analysed, in relation to structures of $\left[A_{i j}\right]_{+}$and the sets of structures of $\omega_{i} \omega_{i}$ and $S_{i j} S_{i j}$, respectively. Concerning structures of $Q$ and $\left[A_{i j}\right]_{+}$surrounding those of $Q$ itself, it is found that a large proportion of structures of $\left[A_{i j}\right]_{+}$appears much closer with regions either intersecting or at less than one characteristic diameter of the tube-like structures of $Q$, and covering a larger proportion of their area than other surrounding structures of $Q$. These are, on average, farther than five diameters apart from themselves, as is also a second group of $\left[A_{i j}\right]_{+}$structures, which might be surrounding those other $Q$ structures at a closer distance.

Regarding structures of $\omega_{i} \omega_{i}$ at an intermediate scale (scale number 3), which were found to be predominantly tube-like in the previous geometrical study ( $(2)$, we have considered the surrounding structures at the same and smaller scales (i.e. scale numbers from 3 to 6) of both $\omega_{i} \omega_{i}$ and $S_{i j} S_{i j}$, each field independently. In the first case, the set of structures of $\omega_{i} \omega_{i}$ at scale number 4 is the predominant group surrounding tubes of $\omega_{i} \omega_{i}$ at scale number 3. Structures of smaller scales (i.e. scale numbers 5 and 6) are also found close to those at scale number 3. In comparison, structures at scale number 3 appear farther among themselves. Concerning geometries of proximal structures, we find two predominant groups: sheet-like structures, which appear closer on average, and tube-like structures, certainly farther but with a high proportion of the total area coverage. Other geometries, intermediate between tubes and sheets, also had a contribution, but to a lesser degree of both proximity and area coverage.

In the second case, when structures of $S_{i j} S_{i j}$ at scale numbers 3-6 surrounding structures of $\omega_{i} \omega_{i}$ at scale number 3 are studied, all scales show similar results of proximity and area coverage. Structures at scale number 3 of $S_{i j} S_{i j}$ have a slightly higher value of proximity, likely owing to the similarity of some structures of both $\omega_{i} \omega_{i}$ and $S_{i j} S_{i j}$ at that intermediate scale. Regarding the geometry of proximal structures in this case, they are primarily sheet-like with significantly higher proximity values and area coverage. Other geometries can be seen among the surrounding structures, but they tend more towards the region of blob-like structures, instead of tube-like structures, as opposed to the case of surrounding structures of $\omega_{i} \omega_{i}$ itself. Also, these other geometries are found farther and cover a smaller area fraction, on average.

Physically, these findings are consistent with the phenomenology of tubes being generated by one or multiple proximal sheets discussed in $\$ 2.6$. Furthermore, as Ruetsch \& Maxey (1992) pointed out when studying the evolution of smallscale structures in incompressible homogeneous isotropic turbulence, vortex tubes and vortex sheets should not be considered as separate, independent structures. 
Instead, they form composite structures, as those shown in figure 27 , with dependent geometries.

The results of this proximity analysis also support the structure-based explanation of intermittency, previously explored by Moisy \& Jiménez (2004) using box counting methods. They found that intense structures form clusters of inertial-range extent. In our case, the low values of the cumulative marginal pdfs encountered for intermediate values of proximity suggest the existence of empty regions (in the iso-contour context) in physical domain in between composite structures.

\section{Conclusions}

In this paper, we studied first the geometry of structures of two scalar fields, $\omega_{i} \omega_{i}$ and $S_{i j} S_{i j}$, from a DNS of incompressible homogeneous isotropic turbulence decaying in time in a periodic box, at the time of maximum enstrophy of the flow. Three different grid resolutions were analysed, corresponding to $256^{3}, 512^{3}$ and $1024^{3}$ points, with identical initial conditions and similar $R e_{\lambda} \approx 77$, resulting in $k_{\max } \bar{\eta}$ of approximately 1,2 and 4, respectively. This allowed us to compare the geometry of the structures for different resolutions and evaluate whether the traditional DNS grid-resolution criterion $k_{\max } \bar{\eta} \approx 1$ is adequate for such geometrical analysis of the educed structures.

The $1024^{3}$ case showed a continuous transition, for decreasing scale, from blob-like and moderately stretched tube-like structures at large scales to highly stretched sheetlike structures at the smallest scales under study. Intermediate scales of $\omega_{i} \omega_{i}$ show a dominance of tube-like structures, which is consistent with the presence of so-called 'worms' in previous studies (Siggia 1981; Jiménez et al. 1993). Tube-like structures also appear at intermediate scales of $S_{i j} S_{i j}$, but in less proportion than for $\omega_{i} \omega_{i}$. The case with smallest grid resolution $\left(256^{3}\right.$ points $)$ did not capture the predominance of highly stretched sheet-like structures educed for the small scales at higher grid resolutions. This suggests the necessity to resolve sub-Kolmogorov scales for a proper geometrical study of the smallest structures of intermittent fields in turbulence, as stated in the literature (see Schumacher et al. 2005; Horiuti \& Fujisawa 2008).

For the $1024^{3}$ case, clustering techniques used during the classification step to obtain distinct groups of geometries among the educed structures resulted in three and two as the optimum number of groups obtained for $\omega_{i} \omega_{i}$ and $S_{i j} S_{i j}$, respectively. Blobs, tubes and sheets can be seen as the predominant structures in the three groups of $\omega_{i} \omega_{i}$, while blobs and sheets are predominant in $S_{i j} S_{i j}$, but tubes, present also in this latter field, were included among the two optimum groups. Optimality scores for other number of groups did not differ substantially from the optimal results. This is a consequence of the continuous distribution of geometries, which indicates that the educed groups are not highly differentiated from each other and that the clustering results in this case should be considered with reserve.

Second, we applied the same non-local methodology for the study of the geometry of structures to two scalar fields, $Q$ and $\left[A_{i j}\right]_{+}$, used by local criteria of identification of tubes and sheets in turbulence, based on the physical meaning of those quantities, that have been proposed in the fluid mechanics literature. This application confirmed the geometrical character expected for the majority of structures educed from those two fields (which before had been done only visually) by providing the necessary mathematical and geometrical background as well as means for quantifying the frequency of appearance of each geometry. Clustering techniques in this case provided a much clearer optimum number of two groups of structures, well differentiated. 
Ninety-six percent of the structures of $Q$ and $\left[A_{i j}\right]_{+}$were assigned to separate groups by the clustering algorithm. $Q$ structures were found mainly to be tube-like, while $\left[A_{i j}\right]_{+}$were recognized as sheet-like. A small amount of structures of both fields presents a geometry that does not correspond to the expected shape. For example, some tubes were found among structures of $\left[A_{i j}\right]_{+}$.

Finally, we introduced a new methodology for the study of proximity issues among structures corresponding to different fields, from a geometrical perspective. It provides information about the type of geometry found in structures of one group surrounding those of another, indicating the proximity and area coverage, by means of joint probability density functions. The set of surrounding structures can also be split into groups, and quantitative results for each group, concerning the proximity to the other structures, are shown by means of cumulative marginal probability density functions. The representation of the geometrical character of each structure is closely related to the visualization space used in the classification step of the previous study of the geometry of structures in turbulence (as introduced in BP). We applied this new technique to structures of $Q,\left[A_{i j}\right]_{+}, \omega_{i} \omega_{i}$ and $S_{i j} S_{i j}$, taken by pairs.

Structures of $Q$ appear closely surrounded, partially overlapped and/or intersected by those of $\left[A_{i j}\right]_{+}$. Comparatively, other structures of $Q$ appear farther from themselves and cover a smaller proportion of their area. A second group of proximal structures of $\left[A_{i j}\right]_{+}$surrounds those of $Q$ at a farther distance, comparable to the distances where other structures of $Q$ are located, which they might be closely surrounding.

Considering only structures of $\omega_{i} \omega_{i}$, those extracted at an intermediate scale (predominantly tube-like) are surrounded primarily by $\omega_{i} \omega_{i}$ structures at the immediately smaller scale, and to a lesser degree by structures of even smaller scales. Structures of $\omega_{i} \omega_{i}$ at the same intermediate scale appear significantly farther. Two groups of surrounding geometries are significant: sheet-like structures are closer; tube-like structures are farther, but they cover a large proportion of the area of the structures they surround, thus indicating that the close sheet-like structures are not eclipsing them.

When $\omega_{i} \omega_{i}$ at the same intermediate scale are studied in relation to the structures of $S_{i j} S_{i j}$ at that and smaller scales, a more balanced contribution from all scales is observed. Sheet-like geometries are again the closest, and they appear to wrap around the tubes of $\omega_{i} \omega_{i}$, eclipsing more effectively other farther geometries.

Several configurations of composite structures were presented. They were associated with different degrees of proximity described above. In particular, the configuration of sheets closely surrounding tubes is consistent with spiral-like super-structures found in the formation process of vortex tubes by rolling-up of sheets. Another common configuration consists of tubes intersected by more planar structures that follow their spatial extent and often connect to other tubes with similar orientations. The grouping of proximal individual structures to form these composite structures reflects the intermittent character of the fields under study, by which empty regions appear among those composite structures. This effect is propagated to the smaller scales and is shown through the marginal cumulative pdfs in terms of proximity.

The general framework of this new methodology, for the study of proximity issues among structures, allows the incorporation of more geometrical and non-geometrical parameters in the analysis (by increasing the size of the array of parameters used in the computation of the conditional array map). Inclusion of physical properties associated with each structure could be helpful when studying how structures of one field are dynamically affected by those of other fields. In this context, tracking the 

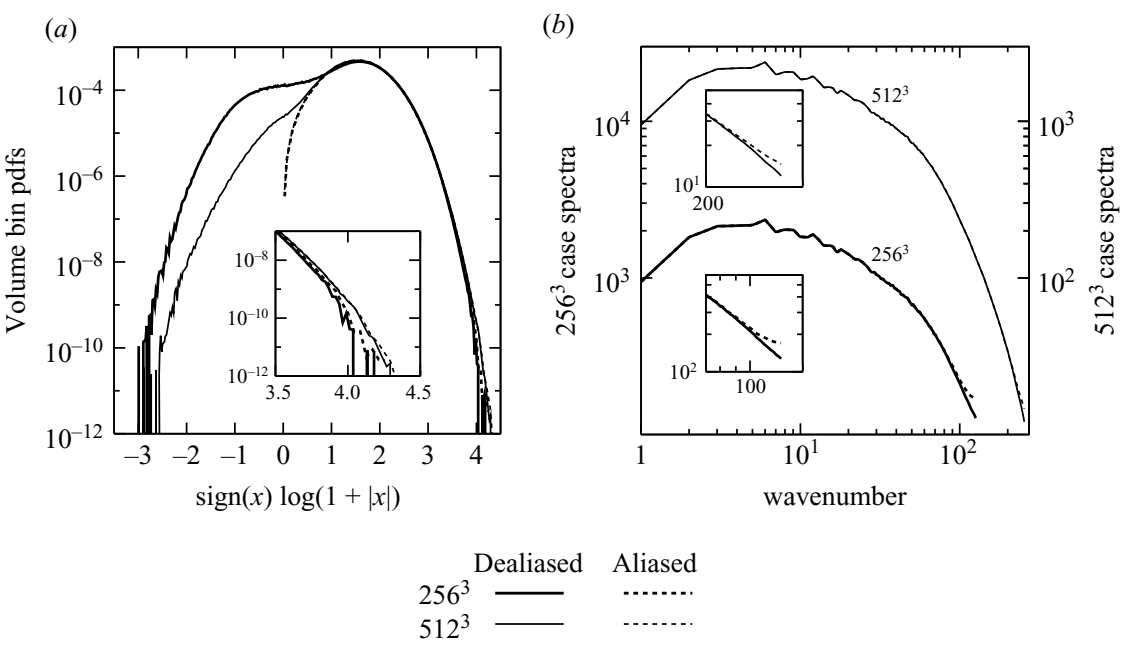

FIGURE 30. Volume pdfs in physical domain $(a)$ and spectra in Fourier domain $(b)$ of aliased and dealiased $S_{i j} S_{i j}$ fields for $256^{3}$ and $512^{3}$ grid resolutions. Note that the volume pdfs use a transformation of the form $\operatorname{sign}(x) \log (1+|x|)$ in the abscissa coordinate. The spectra use two different vertical axes, one for the $256^{3}$ case and the other for the $512^{3}$ case, shifted one decade with respect to each other for a clear view of all curves. The plot of the pdfs includes an inner subplot with a zoomed view of the right tails. The plot of the spectra contains two subplots with zoomed views of the right tails of the $256^{3}$ case (bottom) and $512^{3}$ case (top).

time evolution of the geometrical and proximity properties of these structures, at the individual and composite level, would also expand our understanding of their inter-dependent dynamics.

This work has been supported in part by the National Science Foundation under Grant FRG DMS-0353838. K. H. is supported by Grants-in-Aid from the Ministry of Education, Culture, Sports, Science and Technology, Japan (No. 18560156). Part of the computations were performed at Cybermedia Centre, Osaka University and National Institute for Fusion Science.

\section{Appendix A. Effect of aliasing error}

Even though the velocity field, $u_{i}$, is free of aliasing error, and so are $S_{i j}$ and $\omega_{i}$, the computation of the products of its components in physical space introduces aliasing error in the resulting $S_{i j} S_{i j}$ and $\omega_{i} \omega_{i}$ fields. This error can be removed by means of the 3/2-rule to obtain a dealiased field.

We show a comparison of the analysis of aliased and dealiased $S_{i j} S_{i j}$ fields in the $256^{3}$ and $512^{3}$ grid resolution cases. This allows an evaluation of the effect of aliasing error in the educed geometries for this particular database. The same comparison was performed for the $\omega_{i} \omega_{i}$ field, finding equivalent results (not shown).

Figure 30 shows the volume pdfs and spectra of aliased and dealiased $S_{i j} S_{i j}$ fields, for both $256^{3}$ and $512^{3}$ cases. It is observed, by looking at the spectra, that aliasing error introduces small differences at the end of each spectrum. The difference is more significant for the $256^{3}$ case. Therefore, it is expected that aliasing error will mainly affect the smallest scale of each case, since the spectra of larger scales are the same for the aliased and dealiased fields. While removing the aliasing error improves the spectrum, it also introduces negative values in the resulting dealiased $S_{i j} S_{i j}$ field, as it 


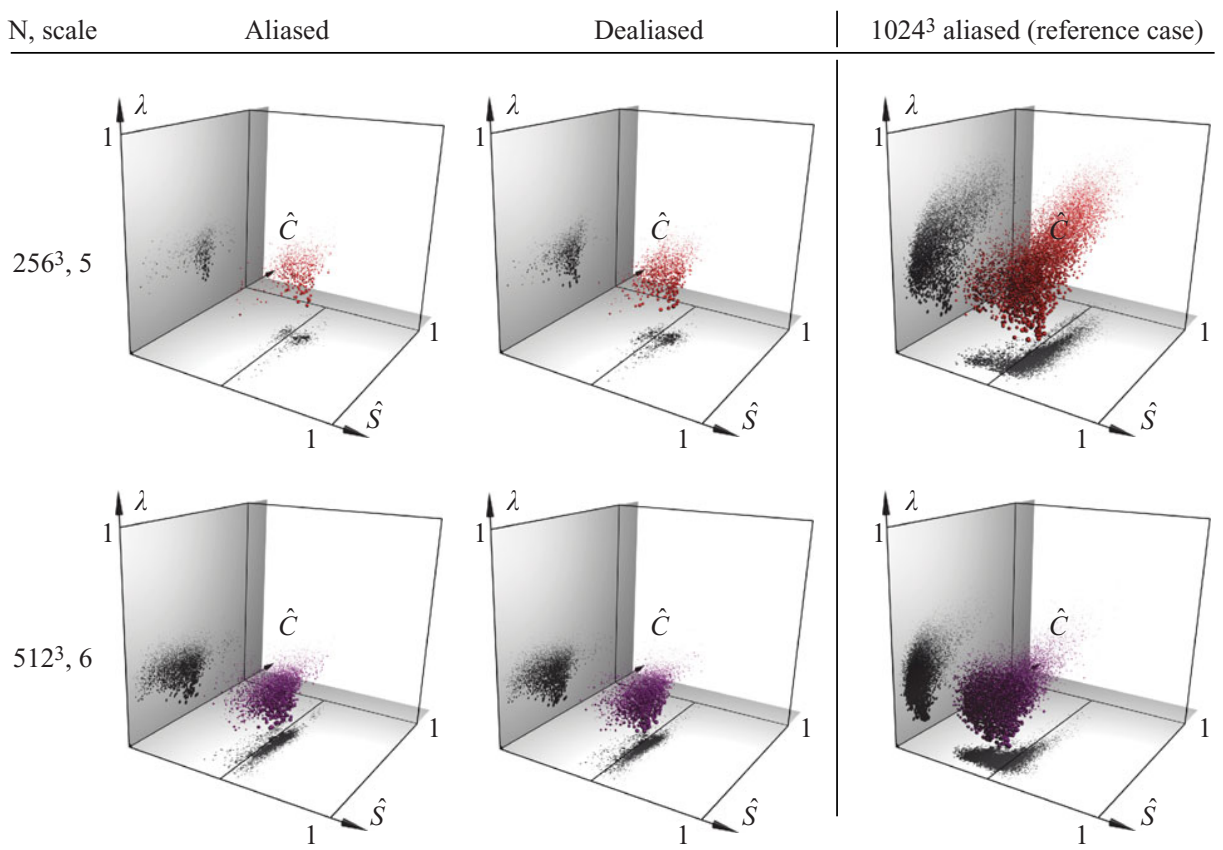

FIGURE 31. Three-dimensional views of the visualization spaces, with glyphs (spheres) representing educed structures of the aliased and dealiased $S_{i j} S_{i j}$ fields, for the smallest scales of the $256^{3}$ (scale 5) and $512^{3}$ (scale 6) grid resolution cases. On the right, the visualization space corresponding to the $1024^{3}$ grid resolution case is shown for reference. Glyphs are scaled by the lognormalized area of the corresponding structure.

can be observed in the volume pdfs. This effect is more significant for the $256^{3}$ case. We note that $S_{i j} S_{i j}$ is, by definition, a positive quantity, so those negative values are non-physical, and result from the application of the 3/2-rule.

Figure 31 shows a comparison of the three-dimensional views of the visualization spaces containing the glyphs representing the geometry of the structures educed from the aliased and dealiased $S_{i j} S_{i j}$ fields at the smallest scales of the $256^{3}$ and $512^{3}$ grid resolution cases (scale numbers 5 and 6 , respectively). On the right, the visualization spaces corresponding to the $1024^{3}$ grid resolution case at the same scales (5 and 6) are shown for comparison purposes. Note that the aliasing error of the $1024^{3}$ case used for reference would affect scale number 7, while scale numbers 5 and 6 will be practically unaffected, and thus it is legitimate to use it for comparison with smaller resolution cases. It is observed, particularly for the $256^{3}$ case, that a slightly higher number of structures of appreciable size was educed when aliasing error was removed, compared to the aliased field. Nonetheless, the number and, especially, the location of these new structures in the visualization space does not significantly improve the results when compared to the reference case: glyphs in the $256^{3}$ case appear with intermediate values of $\hat{C}$, while there is a high concentration of glyphs with low values of $\hat{C}$ in the reference case that are not educed even when aliasing error is removed. The same comments apply to the $512^{3}$ case at scale 6 , when compared to the reference case, which again shows a higher concentration of glyphs with low $\hat{C}$.

In summary, removing the aliasing error in this case did not significantly improve the geometrical results for the smallest scale, while it did modify the volume pdfs in 
physical space, adding negative non-physical values. As expected, by looking at the spectra of figure 30, the geometrical analysis for structures educed at larger scales (larger than the smallest scale attainable for each grid resolution) was found to be practically identical for the aliased and dealiased fields.

\section{Appendix B. Stratified sampling with disproportionate allocation}

When a multi-scale decomposition is applied to the scalar field from which the structures are educed, large differences among the number of structures obtained for each scale are expected. Larger scales will generally have a smaller number of educed structures than smaller scales. This difference in number can sometimes be of several orders of magnitude, particularly when analyzing fields with a high grid resolution, that results in a larger number of scales.

Thus, when structures of all scales are considered in the clustering algorithm, after the geometrical characterization, those structures (and their geometries) corresponding to the largest scale can be under-represented, owing to the much smaller population they have, compared to the others. In such scenario, it can be beneficial to apply, prior to the clustering algorithm itself, a sampling of the population that takes into account the uneven sizes of the strata in which it can be divided.

We use a disproportionate stratification that considers the variance of the mutually exclusive strata to determine the sample size for each stratum. If $n_{o}$ is the sample size of the stratum with the minimum standard deviation, $\sigma_{o}=\min \left\{\sigma_{h}, \forall h\right\}$, then the sample size, $n_{h}$, of any other stratum, $h$, with standard deviation $\sigma_{h}$ will be proportional to $\left(\sigma_{h} / \sigma_{o}\right) n_{o}$. Therefore, those strata with higher variances will also have a higher number of elements to represent them in the clustering algorithm, accounting for their higher diversity. We take $n_{o}$ as the population size of that stratum with the minimum standard deviation, $N_{o}$, since the purpose of this sampling is not to reduce the global population size, but to have a more balanced representation of the different groups present in it for a better clustering.

After the disproportionate stratification, for those strata with $n_{h}<N_{h}$, where $N_{h}$ is the population size of the stratum $h$, we take a random sample of $n_{h}$ out of the $N_{h}$ elements. Otherwise, the complete population is considered for that stratum.

\section{Appendix C. Example of application of proximity algorithm}

Consider a three-dimensional pattern of structures formed by: $(i)$ a circular cylinder of length $L$ and radius $R_{c y l i n d e r}$ with cylindrical axis coincident with the $z$ axis, centred at $z=0$; (ii) eight spheres of radius $R_{\text {sphere }}$ and centres $\left( \pm D_{\text {sphere }}, 0, \pm H\right)$ and $\left(0, \pm D_{\text {sphere }}, \pm H\right)$ and (iii) four segments of circular cylindrical sheets, coaxial with the cylinder, of length $L$ and thickness $t$, centred at $\left( \pm D_{\text {sheet }}, 0,0\right)$ and $\left(0, \pm D_{\text {sheet }}, 0\right)$, each covering an azimuthal angle of $(\pi / 2)-2 \gamma$. The transition to the planar caps of the cylinder and the borders of the sheets is rounded with radius $t / 2$. This pattern is depicted schematically in figure 32 up to symmetries. Note that the planes $x=0$, $y=0, z=0$ and $x= \pm y$ are planes of symmetry.

Repetition of this pattern at distances $\left\{ \pm k \cdot D_{\text {cylinder }}, k=1,2,3, \ldots\right\}$ in the $x$ and $y$ directions results in a set of cylinders surrounded by spheres, sheets and other cylinders.

We will apply the methodology introduced in $\S 4.2$ to evaluate how the set of cylinders is surrounded by the set of spheres, sheets and other cylinders, in terms of proximity and area coverage. Thus, $\mathscr{A}$ will denote the set of cylinders and $\mathscr{B}$ will be 
(a)



(b)



FIGURE 32. Schematic views, up to symmetries, of the unitary pattern of a cylinder surrounded by spheres and circular cylindrical segments of sheets. $(a)$ cut by the plane $z=H$ (top view); (b) cut by the plane $y=0$ (front view). Note that $x=0, y=0, z=0$ and $x=y$ are planes of symmetry, and only one-eighth and one-quarter of the pattern are represented on the left and right plots, respectively.

the whole set of structures (cylinders, spheres and sheets). Note that $\mathscr{A} \subset \mathscr{B}$. Here $\mathscr{B}$ can be split in three groups, each with a different group index $g:$ spheres $(g=1)$, sheets $(g=2)$ and cylinders $(g=3)$.

\section{C.1. Area coverage}

As a side problem, consider two co-planar circumferences, $C_{1}$ and $C_{2}$, of radii $R_{1}$ and $R_{2}$, respectively, whose centres are located at a distance $D$ (see figure 33). Take a point $Q^{\prime} \in C_{2}$, defined by an angle $\alpha_{2}^{\prime}$. Define $P^{\prime} \in C_{1}$ such that the distance to $Q^{\prime}$ is minimum among all points of $C_{1}$. The angle $\alpha_{1}^{\prime}$ that defines $P^{\prime}$ is given by $\tan \alpha_{1}^{\prime}=\rho \sin \alpha_{2}^{\prime} /\left(1-\rho \cos \alpha_{2}^{\prime}\right)$, where $\rho=R_{2} / D$. The angle $\alpha_{2}^{\prime}$ for which $\alpha_{1}^{\prime}$ is maximum is given by $\cos \alpha_{2}=\rho$, resulting $\sin \alpha_{1}=\rho$. The points $P^{\prime}$ and $Q^{\prime}$ given by such $\alpha_{1}$ and $\alpha_{2}$ are denoted by $P$ and $Q$. The distance between $P$ and $Q$ is given by $d_{P Q}=\left(D^{2}+R_{1}^{2}+R_{2}^{2}-2 R_{1} \sqrt{1-R_{2}^{2}}-2 R_{2}^{2}\right)^{1 / 2}$. Thus, the arclength of $C_{1}$ covered by $C_{2}$ in the sense of minimum distances is given by $R_{1} \cdot\left(2 \alpha_{1}\right)$, where the factor 2 results from the symmetry of the geometry with respect to the line joining the centres.

Let us translate the previous results obtained for the case of the two circumferences into the three-dimensional problem of cylinders, spheres and sheets. For the pair cylinder-sphere, the angle $\alpha$ (see figure 32) of the cylinder defining the arclength coverage by the maximal circumference of the sphere will be $\alpha=\arcsin \left(R_{\text {sphere }} / D_{\text {sphere }}\right)$. For any other circumference of the sphere (not maximal), $\alpha(r)=\arcsin \left(r / D_{\text {sphere }}\right)$, where $r$ is the radius of the circumference intersection of the sphere and the plane normal to $z$ at a distance $r$ above/below its centre. Thus, the area of the cylinder covered by a sphere (in the minimum distance sense) will be $A C_{\text {sphere }}=2 \int_{0}^{R_{\text {sphere }}} R_{\text {cylinder }} 2 \alpha(r) \mathrm{d} r=4 D_{\text {sphere }} R_{\text {sphere }}\left[\sigma \alpha(\sigma)+\sqrt{1-\sigma^{2}}-1\right]$, where $\sigma=R_{\text {sphere }} / D_{\text {sphere }}$. The factor 2 in front of the integral results from the symmetry of the sphere for $z$-planes above and below the one crossing its centre. 


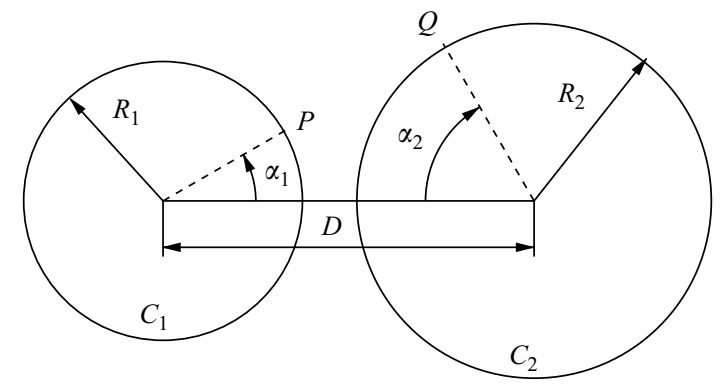

FigURE 33. Length-coverage problem of two circumferences.

Consider now the pair of cylinders with axes $(0,0, z)$ and $\left(D_{c y l i n d e r}, D_{c y l i n d e r}, z\right)$, the latter resulting from the repetition of the unitary pattern at a distance $D_{\text {cylinder }}$ in the $x$ and $y$ directions. They are separated by a distance $\sqrt{2} D_{\text {cylinder }}$. The angle $\gamma$ defining half the arclength coverage of one cylinder to the other (corresponding to the angle $\alpha_{1}$ in the two circumference problem) is $\gamma=\arcsin \left(R_{\text {cylinder }} / \sqrt{2} D_{\text {cylinder }}\right)$. Thus, the area of one cylinder covered by the other (in the minimum distance sense) will be $A C_{\text {cylinder }}=R_{\text {cylinder }} 2 \gamma L$.

We choose the segment of cylindrical sheet in figure 32 to extend up to an azimuthal angle of $\pi / 4-\gamma$. Extending the sheet by symmetry to the $-y$ region, the area coverage of one sheet to its co-axial cylinder will be, in the absence of any other structures, $A C_{\text {sheet }}=R_{\text {cylinder }} 2(\pi / 4-\gamma) L$. With this choice of geometry, only the spheres and sheets in the unitary pattern of a reference cylinder, and the cylinders with axes at a distance $\sqrt{2} D_{\text {cylinder }}$ from it will contribute to its area coverage of that reference cylinder. Other spheres, sheets and cylinders will be eclipsed from it by closer structures and will not contribute to its area coverage. Therefore, for each cylinder of surface area $A_{c y l i n d e r}$,

(a) Area coverage by spheres: $a_{\text {spheres }}=8 A C_{\text {sphere }} / A_{\text {cylinder }}$;

(b) Area coverage by sheets: note that the spheres of the previous item are eclipsing the sheets, as they are located in between the reference cylinder and the sheets, so their contribution needs to be subtracted. Therefore, $a_{\text {sheets }}=4 A C_{\text {sheets }} / A_{\text {cylinder }}-a_{\text {spheres }}$ and

(c) Area coverage by other cylinders: by repetition of the pattern in the $x$ and $y$ coordinates, there will be only four other cylinders surrounding each cylinder that are not eclipsed by sheets or spheres. These correspond to the cylinders at a distance $\sqrt{2} D_{c y l i n d e r}$ from the reference one. Therefore, $a_{c y l i n d e r s}=4 A C_{c y l i n d e r} / A_{c y l i n d e r}$.

\section{C.2. Proximity values}

The local proximity value, $p$, is defined in terms of the distance, $d$, between points of a target and reference structures as $p=(1+d / D)^{-1}$, where $D$ is a non-dimensionalizing distance of the reference structure, chosen as $D=\mu \equiv 3 V / A$, where $V$ is the volume and $A$ is the surface area of the reference structure.

The minimum and maximum proximity values in the conditional array map obtained for each surrounding structure to the reference cylinder, $C A M$ (cylinder), will be

(a) proximity to spheres

$$
\begin{aligned}
& \left.p_{\text {spheres }}^{\text {min }}\right|_{C A M(\text { cylinder })}=\left[1+\left(D_{\text {sphere }}-R_{\text {cylinder }}\right) / \mu_{\text {cylinder }}\right]^{-1}, \\
& \left.p_{\text {spheres }}^{\text {max }}\right|_{C A M(\text { cylinder })}=\left[1+\left(D_{\text {sphere }}-R_{\text {cylinder }}-R_{\text {sphere }}\right) / \mu_{\text {cylinder }}\right]^{-1} ;
\end{aligned}
$$

(b) proximity to sheets

$$
\left.p_{\text {sheets }}^{\min }\right|_{C A M(\text { cylinder })}=\left[1+d^{\prime}\left(t / 2, t / 2, D_{\text {sheet }}-R_{\text {cylinder }}+t / 2\right) / \mu_{\text {cylinder }}\right]^{-1},
$$




$\begin{array}{lccccccc}\text { Structures } & g & \hat{S} & \hat{C} & a & p^{\text {min }} & p^{\text {max }} & p^{\text {mean }} \\ \text { Spheres } & 1 & 1.0 & 1.0 & 0.3 & 0.5 & 0.8 & 0.7 \\ \text { Sheets } & 2 & 0.5 & 0.0 & 0.6 & 0.3 & 0.3 & 0.3 \\ \text { Cylinders } & 3 & 0.5 & 1.1 & 0.1 & 0.1 & 0.1 & 0.1\end{array}$

TABLE 3. Approximate numerical values for each group of structures, $g:(\hat{S}, \hat{C})$ feature centre (in terms of the absolute value of the shape index, $S$, and the dimensionless curvedness, $C) ; a$, area coverage in the proximity sense to the reference cylinder and $\left\{p^{\text {min }}, p^{\text {max }}, p^{\text {mean }}\right\}$, minimum, maximum and mean proximity values from CAM (cylinder).


FIGURE 34. Histograms approximating $\left[\left.\overrightarrow{\mathscr{P} \mathscr{I}}(\xi, \zeta ; p)\right|_{\mathscr{A} \leftarrow \mathscr{B}}(a)\right.$ and $\left[\left.\mathscr{P}(p, g)\right|_{\mathscr{A} \leftarrow \mathscr{B}}(b)\right.$ for the numerical application.

$$
\left.p_{\text {sheets }}^{\text {max }}\right|_{\text {CAM (cylinder })}=\left[1+\left(D_{\text {sheet }}-R_{\text {cylinder }}-t / 2\right) / \mu_{\text {cylinder }}\right]^{-1} \text { and }
$$

(c) proximity to cylinders

$$
\begin{aligned}
& \left.p_{\text {cylinders }}^{\text {min }}\right|_{C A M(\text { cylinder })}=\left[1+d^{\prime}\left(R_{\text {cylinder }}, R_{\text {cylinder }}, \sqrt{2} D_{\text {cylinder }}\right) / \mu_{c y l i n d e r}\right]^{-1}, \\
& \left.p_{\text {cylinders }}^{\text {max }}\right|_{C A M(\text { cylinder })}=\left[1+\left(\sqrt{2} D_{\text {cylinder }}-2 R_{\text {cylinder }}\right) / \mu_{c y l i n d e r}\right]^{-1},
\end{aligned}
$$

where $d^{\prime}(x, y, z) \equiv\left(x^{2}+y^{2}+z^{2}-2 x \sqrt{1-y^{2}}-2 y^{2}\right)^{1 / 2}$ was defined for the twocircumference problem as $d_{P Q}\left(R_{1}, R_{2}, D\right)$. Owing to the geometrical configuration of the unitary pattern of structures, the results of area coverage and proximity obtained for one reference cylinder can be extended to the set $\mathscr{A}$ of all cylinders.

\section{C.3. Numerical application}

Taking $R_{\text {cylinder }}=1, L=10, R_{\text {sphere }}=1.25, D_{\text {sphere }}=2.5, D_{\text {sheet }}=4, D_{\text {cylinder }}=8.2$ and $t=0.1$, then $A_{\text {cylinder }} \approx 69.1, V_{\text {cylinder }} \approx 31.4, \mu_{\text {cylinder }} \approx 1.36$ and table 3 can be obtained based on the previous analysis. From the values on that table, the histograms approximating $\left[\left.\overrightarrow{\mathscr{P}} \mathscr{I}(\xi, \zeta ; p)\right|_{\mathscr{A} \leftarrow \mathscr{B}}\right.$ and $\left[\left.\mathscr{P}(p, g)\right|_{\mathscr{A} \leftarrow \mathscr{B}}\right.$ can be plotted.

Figure $34(a)$ shows the histogram that approximates $\left[\left.\overrightarrow{\mathscr{P} \mathscr{I}}(\xi, \zeta ; p)\right|_{\mathscr{A} \leftarrow \mathscr{B}}\right.$, with the $\hat{S}$ and $\hat{C}$ divided into 32 and 40 intervals, respectively. There are only three twodimensional intervals (squares) contributing to the histogram. The location of those squares corresponds to the feature centre $(\hat{S}, \hat{C})$ of each type of surrounding structure (see table 3$):(1,1)$ for spheres, $(0.5,0)$ for sheets and $(0.5,1.1)$ for cylinders. The 
colour of each square corresponds to the mean proximity value for the corresponding structures associated with that square: the red square, corresponding to the spheres, has the highest mean proximity, 0.7 ; the green one corresponds to the sheets, with an intermediate mean proximity of 0.3 and the blue square, corresponding to the other cylinders, has a low mean proximity of 0.1 . The higher the saturation of the colour of each square, the higher the area coverage of that type of structures; in this case, we found an area coverage of 0.3 for the spheres, 0.6 for the sheets and 0.1 for the cylinders, so the highest saturation will be that of the green square, and the lowest saturation corresponds to the blue square, while the red square will have an intermediate saturation.

Figure $34(b)$ shows the histogram that approximates $\left[\left.\mathscr{P}(p, g)\right|_{\mathscr{A} \leftarrow \mathscr{B}}\right.$, with the proximity $p$ axis divided into 20 intervals. The values of proximity of each group, $g$, correspond to those reflected in table 3: $0.5-0.8$ for spheres, 0.3 for sheets and 0.1 for cylinders. The integral below the pdf for each group corresponds to the value of the area coverage of the cylinders in the proximity sense shown in table 3: 0.3 for spheres, 0.6 for sheets and 0.1 for other cylinders. Note that the histogram for the spheres $(p \in[0.5-0.8])$ has been approximated by a constant value throughout the 7 intervals it spans, $(0.3 /(7 \cdot 0.05))$, for simplicity.

\section{REFERENCES}

Ashurst, W. T., Kerstein, A. R., Kerr, R. M. \& Gibson, C. H. 1987 Alignment of vorticity and scalar gradient with strain rate in simulated Navier-Stokes turbulence. Phys. Fluids 30, 2343-2353.

BAtchelor, G. K. \& Townsend, A. A. 1949 The nature of turbulent motion at large wavenumbers. Proc. R. Soc. Lond. A 199, 238-255.

Bermejo-Moreno, I. \& Pullin, D. I. 2008 On the non-local geometry of turbulence. J. Fluid Mech. 603, 101-135.

Bradshaw, P. \& KoH, Y. M. 1981 A note on Poisson's equation for pressure in a turbulent flow. Phys. Fluids 24, 777.

Brasseur, J. G. \& WANG, Q. 1992 Structural evolution of intermittency and anisotropy at different scales analyzed using three-dimensional wavelet transforms. Phys. Fluids A 4, 2538-2554.

Candès, E., Demanet, L., Donoho, D. \& Ying, L. 2005 Fast discrete curvelet transforms. Multiscale Model Simul. 5, 861-899.

Chen, S., Sreenivasan, K. R. \& Nelkin, M. 1997 Inertial range scalings of dissipation and enstrophy in isotropic turbulence. Phys. Rev. Lett. 79, 1253-1256.

Goto, Susumu 2008 A physical mechanism of the energy cascade in homogeneous isotropic turbulence. J. Fluid Mech. 605, 355-266.

Hamlington, P. E., Schumacher, J. \& Dahm, W. J. A. 2008 Local and nonlocal strain rate fields and vorticity alignment in turbulent flows. Phys. Rev. E 77, 026303.

He, G., Chen, S., Kraichan, R. H., Zhang, R. \& Zhou, Y. 1998 Statistics of dissipation and enstrophy induced by localized vortices. Phys. Rev. Lett. 81, 4639.

Horiuti, K. 2001 A classification method for vortex sheet and tube structures in turbulent flows. Phys. Fluids A 13 (12), 3756-3774.

Horiuti, K. \& FujISAWA, T. 2008 The multi-mode stretched spiral vortex in homogeneous isotropic turbulence. J. Fluid Mech. 595, 341-366.

Horiuti, K. \& TAKagi, Y. 2005 Identification method for vortex sheet structures in turbulent flows. Phys. Fluids 17 (12), 121703.

Hunt, J. C. R., Wray, A. A. \& Moin, P. 1988 Eddies, stream, and convergence zones in turbulent flows. Tech. Rep. Center for Turbulence Research Report CTR-S88.

Ishinara, T., Kaneda, Y., YokoKawa, M., Itakura, K. \& UnO, A. 2003 Spectra of energy dissipation, enstrophy and pressure by high-resolution direct numerical simulations of turbulence in a periodic box. J. Phys. Soc. Japan 72, 983-986.

Jeong, J. \& Hussain, F. 1995 On the identification of a vortex. J. Fluid Mech. 285, 69-94. 
JimÉnEZ, J. 1992 Kinematic alignment effects in turbulent flows. Phys. Fluids A 4, 652654.

Jiménez, J., Wray, A. W., Saffman, P. G. \& Rogallo, R. S. 1993 The structure of intense vorticity in isotropic turbulence. J. Fluid Mech. 255, 65-90.

Kennedy, D. A. \& Corrsin, S. 1961 Spectral flatness factor and intermittency in turbulence and in non-linear noise. J. Fluid Mech. 10, 366-370.

KERR, R. M. 1985 Higher-order derivative correlations and the alignment of small-scale structures in isotropic numerical turbulence. J. Fluid Mech. 153, 31-58.

Koenderink, J. J. \& van Doorn, A. J. 1992 Surface shape and curvature scales. Image Vision Comput. 10 (8), 557-565.

Kolmogorov, A. N. 1941 a Dissipation of energy in a locally isotropic turbulence. Dokl. Nauk. SSSR. 32, 16-18.

Kolmogorov, A. N. $1941 b$ The local structure of turbulence in incompressible viscous fluids at very large Reynolds numbers. Dokl. Nauk. SSSR. 30, 301-305.

Kolmogorov, A. N. 1962 A refinement of previous hypotheses concerning the local structure of turbulence in a viscous incompressible fluid at high Reynolds numbers. J. Fluid Mech. 13, $82-85$.

Kraichnan, R. H. 1974 On Kolmogorov's inertial-range theories. J. Fluid Mech. 62, 305-330.

Landau, L. D. \& Lifshitz, E. M. 1959 Fluid Mechanics. Pergamon.

Lundgren, T. S. 1982 Strained spiral vortex model for turbulent fine structure. Phys. Fluids 25, 2193-2203.

Meneveau, C. 1991 Analysis of turbulence in the orthonormal wavelet representation. J. Fluid Mech. 232, 469-520.

MisRa, A. \& Pullin, D. I. 1997 A vortex-based subgrid stress model for large-eddy simulations. Phys. Fluids 9, 2443-2454.

MoIsY, F. \& JimÉNEZ, J. 2004 Geometry and clustering of intense structures in isotropic turbulence. J. Fluid Mech. 513, 111-133.

Nomura, K. K. \& Post, G. K. 1998 The structure and dynamics of vorticity and rate of strain in incompressible homogeneous turbulence. J. Fluid Mech. 377, 65-97.

Okamoto, N., Yoshimatsu, K., Shneider, K., Farge, M. \& Kaneda, Y. 2007 Coherent vortices in high resolution direct numerical simulation of homogeneous isotropic turbulence: A wavelet viewpoint. Phys. Fluids 19, 115109.

Onsager, L. 1945 The distribution of energy in turbulence. Phys. Rev. 68, 286.

Pullin, D. I. \& Saffman, P. G. 1997 Vortex dynamics in turbulence. Annu. Rev. Fluid Mech. 30, $31-51$.

Richardson, L. F. 1922 Weather Prediction by Numerical Process. Cambridge University Press.

Richardson, L. F. 1926 Atmospheric diffusion shown on a distance-neighbor graph. Proc. R. Soc. Lond. Ser. A 110, 709-737.

Ruetsch, G. R. \& MaXeY, M. R. 1992 The evolution of small-scale structures in isotropic homogeneous turbulence. Phys. Fluids A 4 (12), 2747-2760.

Schumacher, J., Sreenivasan, K. R. \& Yeung, P. K. 2005 Very fine structures in scalar mixing. J. Fluid Mech. 531, 113-122.

SigGia, E. D. 1981 Numerical study of small-scale intermittency in three-dimensional turbulence. J. Fluid Mech. 107, 375-406.

Sreenivasan, K. R. 2004 Possible effects of small-scale intermittency in turbulent reacting flows. Flow, Turbul. Combus. 72, 115-131.

Sreenivasan, K. R. \& Meneveau, C. 1988 Singularities of the the equations of fluid motion. Phys. Rev. A 38, 6287-6295.

Tanaka, M. \& Kida, S. 1993 Characterization of vortex tubes and sheets. Phys. Fluids A 5, 2079-2082.

TaO, B., Katz, J. \& Meneveau, C. 2000 Geometry and scale relationships in high Reynolds number turbulence determined from three-dimensional holographic velocimetry. Phys. Fluids 12, 941-944.

Tsinober, A., Kit, E. \& Dracos, T. 1992 Experimental investigation of the field of velocity-gradients in turbulent flows. J. Fluid Mech. 242, 169-192. 
Vincent, A. \& Meneguzzi, M. 1994 The dynamics of vorticity tubes in homogeneous turbulence. J. Fluid Mech. 258, 245-254.

Wang, L. \& Peters, N. 2006 The length-scale distribution function of the distance between extremal points in passive scalar turbulence. J. Fluid. Mech. 554, 457-475.

Wu, J-Z., Zhou, Y. \& Fan, M. 1999 A note on kinetic energy, dissipation and enstrophy. Phys. Fluids 11, 503-505.

Zeff, B. W., Lanterman, D. D., McAllister, R., Roy, R., Kostelich, E. J. \& Lathrop, D. P. 2003 Measuring intense rotation and dissipation in turbulent flows. Nature 421, 146-149. 